University of Louisville

ThinkIR: The University of Louisville's Institutional Repository

Electronic Theses and Dissertations

$12-2013$

\title{
Post-mortem analysis of cortical thickness and neuronal morphometry in autism.
}

Shweta Sunil Kamat

University of Louisville

Follow this and additional works at: https://ir.library.louisville.edu/etd

\section{Recommended Citation}

Kamat, Shweta Sunil, "Post-mortem analysis of cortical thickness and neuronal morphometry in autism." (2013). Electronic Theses and Dissertations. Paper 722.

https://doi.org/10.18297/etd/722

This Master's Thesis is brought to you for free and open access by ThinkIR: The University of Louisville's Institutional Repository. It has been accepted for inclusion in Electronic Theses and Dissertations by an authorized administrator of ThinkIR: The University of Louisville's Institutional Repository. This title appears here courtesy of the author, who has retained all other copyrights. For more information, please contact thinkir@louisville.edu. 


\title{
POST-MORTEM ANALYSIS OF CORTICAL THICKNESS AND NEURONAL MORPHOMETRY IN AUTISM
}

\author{
By
}

Shweta Sunil Kamat

\begin{abstract}
A Thesis
Submitted to the Faculty of the

University of Louisville School of Medicine

In Partial Fulfillment of the Requirements

For the Degree of
\end{abstract}

Master of Science

Department of Anatomical Sciences and Neurobiology

University of Louisville

Louisville, Kentucky

December 2013 
POST-MORTEM STUDY OF CORTICAL THICKNESS AND NEURONAL MORPHOMETRY IN AUTISM

By

Shweta Sunil Kamat

A Thesis Approved on

November 14, 2013

by the following Thesis Committee:

Dr. Manuel F. Casanova

Dr. David S. Magnuson

Dr. Nobyuki Kuwabara 


\section{ACKNOWLEDGMENTS}

It would not have been possible to complete this project and write my thesis without the help and support of the kind people around me, to only some of whom it is possible to give particular mention here. First, I offer my sincere gratitude to Dr. Manuel Casanova, for granting me the privilege of joining his lab in order to learn about the various aspects of scientific research, and standing by me in my journey as a graduate student. I would also like to thank my committee members, Dr. David Magnuson and Dr. Nobuyuki Kuwabara, for frequently going above and beyond to guide me in the research development and for patiently teaching me throughout the academic coursework. I would like to recognize Dr. Ayman El-baz and his lab members, especially Ahmed Elnakib and Fahmi Khalifa, for assisting in the segmentation process and developing the algorithm utilized to measure cortical thickness. I would also like to thank Andrew E. Switala for his contribution toward the statistics aspect of this project; and Anita Allison-McNutt for her assistance in scanning the images of the coronal slides.

In addition to those who directly contributed to my research pursuits, many faculty members at the UofL School of Medicine have played an essential role in my progression as a student; I would like to offer a special thanks to Dr. Jennifer Brueckner and Dr. Vinay Puri. I thank them for believing in my capabilities, and generously giving me the opportunities to grow as a student and prepare for future

professional aspirations. I would also like to recognize the ASNB department staff for 
their cooperation, especially Barbara Hughes, for patiently helping me to resolve any administrative issues. I would also like to acknowledge my lab colleagues and good friends: Brynn Dombroski and Ryan Kiser, for their peer-mentorship and support since my first day in the ASNB program. I also thank my close friends and family for their ceaseless support and encouragement throughout this process. Most importantly, I would like to extend my deepest gratitude to my parents, Sunil and Nitha Kamat, to whom I owe all my achievements and success; it would not have been possible for me to pursue my goals without their unconditional care and blessings. 


\section{ABSTRACT \\ POST-MORTEM STUDY OF CORTICAL THICKNESS AND NEURONAL MORPHOMETRY IN AUTISM \\ Shweta Sunil Kamat \\ May 25, 2013}

Autism Spectrum Disorders (ASD) are a group of conditions characterized by a broad spectrum of deficiencies related to social communication, both verbal and non-verbal, as well as repetitive behaviors such as rocking back and forth, or arm flapping. This devastating group of disorders affects millions of people regardless of their socioeconomic, ethnic and racial backgrounds. Among other issues such as migraines, sensory deficits and intense aggression, epilepsy is a major neurological disorder very highly associated with ASD; roughly one third of all ASD patients are diagnosed with epilepsy, and a much higher proportion suffer from at least one seizure in their lifetime. Past neuropathological studies have suggested various deformities in the brains of ASD patients, ranging from abnormal brain volume, and head circumference, to the presence of atrophy and cortical dysplasias, such as heterotopias. Other more cellular abnormalities have also been speculated, including neurotransmitter imbalances, as well as neuron proliferation and organization disturbances. Though many intricate techniques have been attempted in order to investigate the etiology and potential treatments of ASD pathology, the results have been inconclusive and highly arguable. This especially applies to magnetic resonance imaging (MRI) studies of cortical thickness, which have produced highly questionable and inconsistent information; researchers have attributed this to the potential instability of the mode of research. Due to the limitations on the resolution 
of MRI, it is difficult to directly compute the location of the pial surface, which creates difficulty in delineating the cortical gray matter from the subcortical white matter. The goal of our study was to indentify cortical dysplasias, and then to analyze the neuromorphology of the same areas. In order to accomplish this, we retrieved postmortem tissue from the Autism Tissue Program, and measured the cortical thickness by solving the Laplace equation, followed by the application of the Boolean model and granulometry to determine the potential abnormalities of ASD neuronal morphometry. We found multiple dysplasias in various brain regions, predominantly within the pre-frontal cortex, which correlated nicely with the symptomology of ASD. The anterior commissure $(\mathrm{AC})$ served as the landmark to delineate the prefrontal cortex; when compared with neurotypical tissue, the ASD tissue was thinner surrounding the AC. Upon further analysis of the dysplastic processes, our findings confirmed the presence of smaller, but more numerous pyramidal neurons in the ASD brain when compared with neurotypicals. These findings are also substantial in terms of explaining two of the more prominent issues associated with ASD: aggressive behavior and frequent seizures. Overall, the findings of the current study do support several previous reports and provides further evidence of problematic cortical development and ASD symptom manifestation. 


\section{TABLE OF CONTENTS}

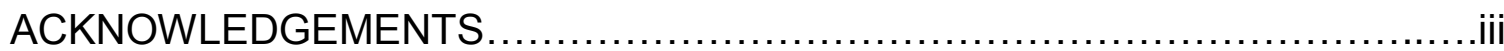

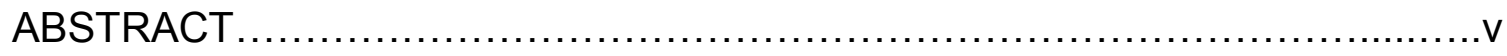

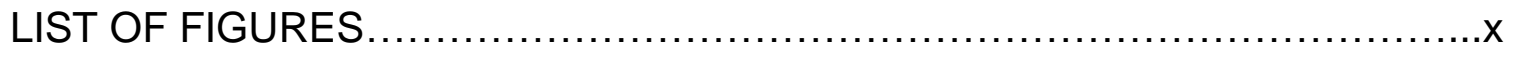

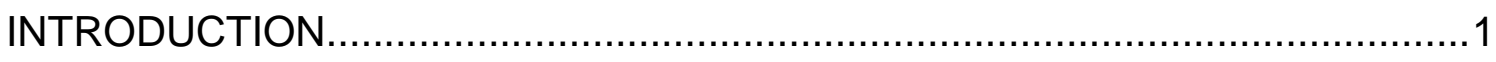

Definition and Diagnosis of

Autism Spectrum Disorder...................................................

Prevalence and Cause...........................................................

Neurobiology and Neuropathology

of Autism Spectrum Disorder..........................................................

Dysplasia vs. Atrophy .................................................................11

Epilepsy/Seizures in Autism..............................................................12

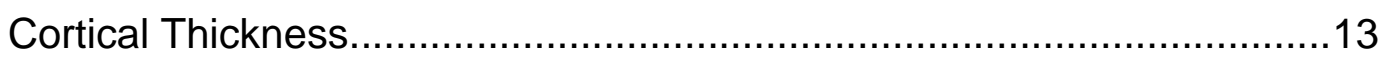

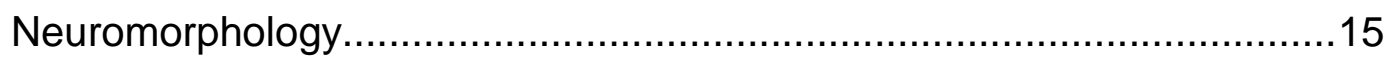

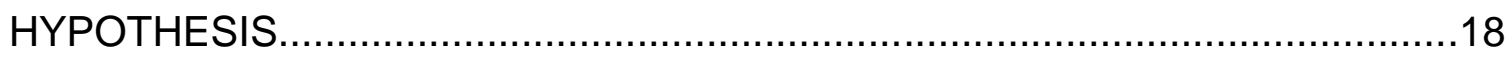

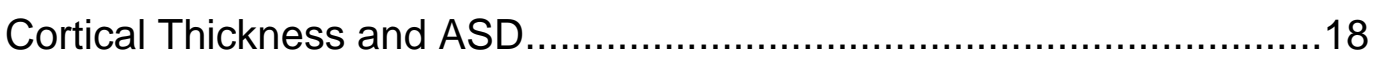

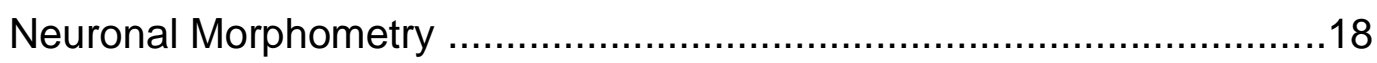

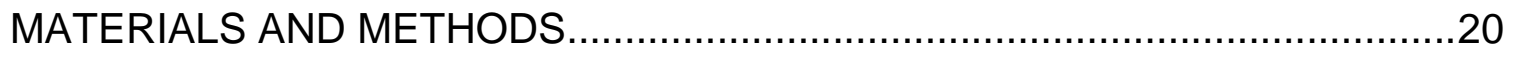

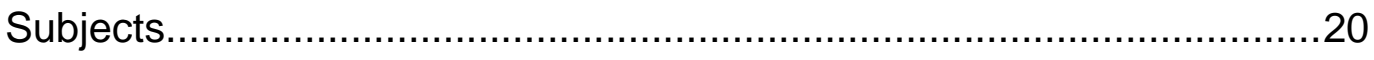

Cortical Thickness Methods...........................................................22

Neuronal Morphometry Methods........................................................26 
RESULTS 30

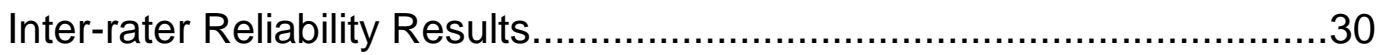

Cortical Thickness Control Vs. Neurotypical............................................

Neuronal Morphometry Control Vs. Neurotypical...................................34

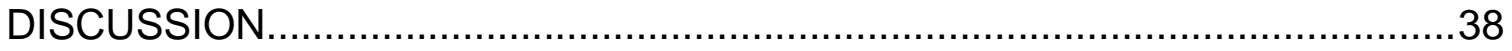

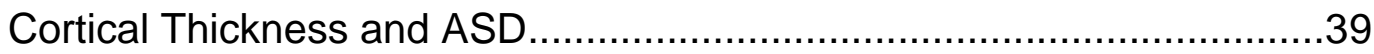

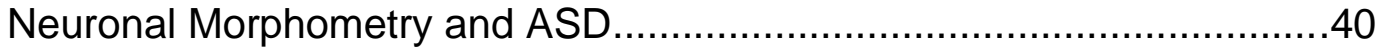

Clinical Significance of Frontal Lobe

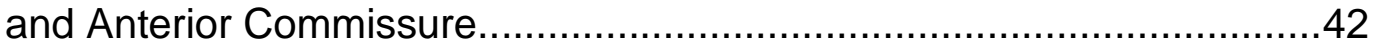

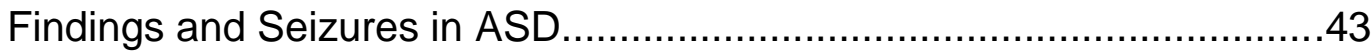

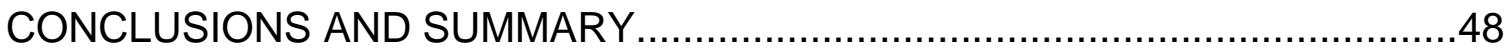

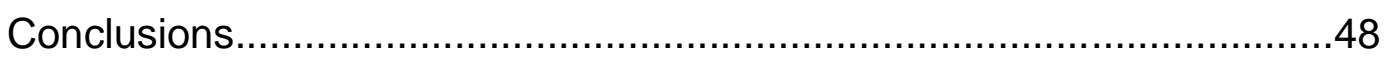

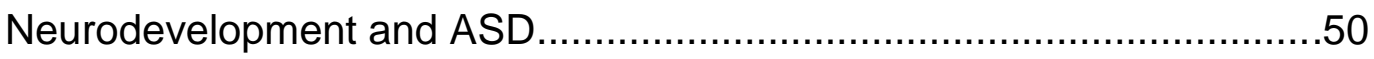

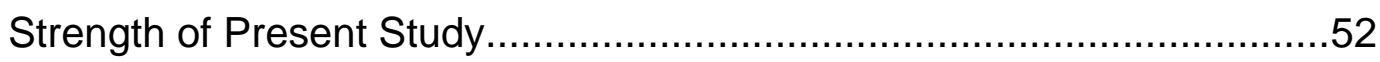

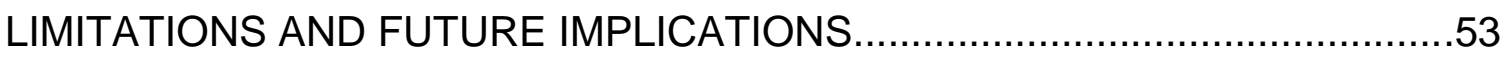

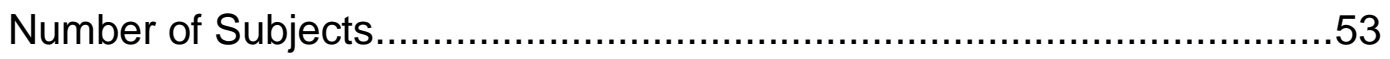

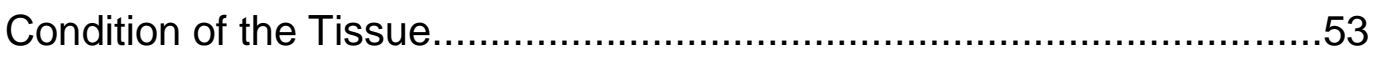

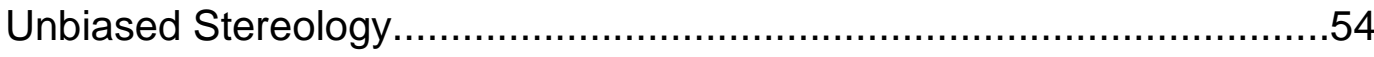

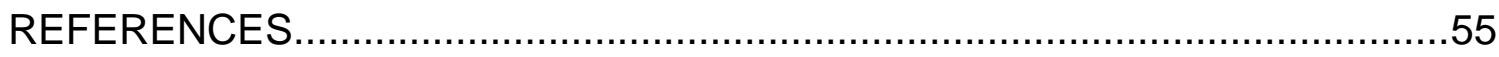

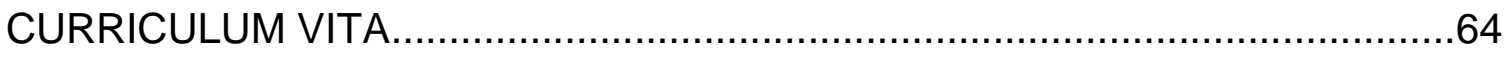




\section{LIST OF FIGURES}

Figure...

Page

1. Scanned images of post mortem tissue:

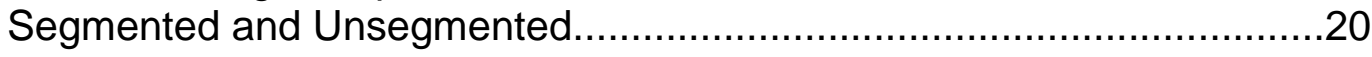

2. Solution of Laplace equation inside the cortical

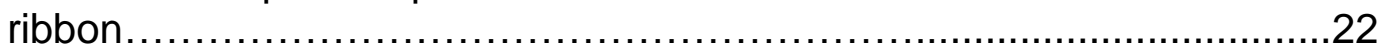

3. Sample photomicrograph of selected region..........................................24

4. Spline smooth graph of differences in cortical

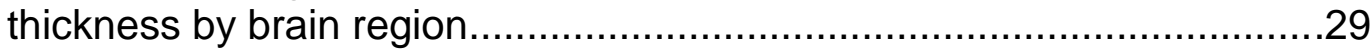

5. Difference in cortical thickness with

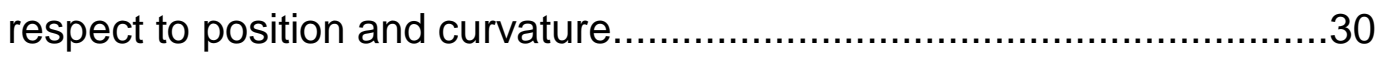

6. Box plot of estimated Boolean model: Mean area..................................32

7. Box plots of estimated Boolean model: Mean perimeter..........................33

8. Granulometry results: Pattern spectrum by lamina.................................34 


\section{INTRODUCTION}

\subsection{Background}

\subsubsection{Definition and Diagnosis of Autism Spectrum Disorder}

Autism spectrum disorder (ASD) is a neurodevelopmental condition that disturbs several day-to-day activities of the affected individuals. According to previous ASD definitions, there were three core symptom domains: deficits in communication, abnormal social interactions, and restrictive and/or repetitive interests and behaviors such as toe-walking, arm flapping, and body rocking (Centers for Disease Control and Prevention, 2012; DiCicco-Bloom, et al., 2006). As per the Diagnostic and Statistical Manual of Mental Disorders, Fourth Edition (DSMIV) the spectrum included Autistic disorder (classic autism), Asperger disorder (Asperger syndrome), Pervasive developmental disorder not otherwise specified (PDD-NOS), Rett disorder (Rett syndrome) and Childhood disintegrative disorder (CDD) (National Institute of Mental Health, 2011).

The recently revised Diagnostic and Statistical Manual on Mental Disorders, Fifth Edition (DSM V), has changed the diagnosis method from the three core symptom domains to now two categories: social communication impairment and restricted interests/repetitive behaviors. More specifically, for a diagnosis of ASD a child must have three deficits in social communication and social interaction (i.e. deficits in social-emotional reciprocity, nonverbal communicative behaviors used for 
social interaction, or in developing and maintaining relationships); and at least two deficits under the restricted, repetitive patterns of behavior, interests, or activities category. The deficits under this criteria include: (1) stereotyped or repetitive speech, motor movements, or use of objects; (2) excessive adherence to routines, ritualized patterns of verbal or nonverbal behavior, or excessive resistance to change; (3) highly restricted, fixated interests; and (4) hyper- or hypo-reactivity to sensory input or unusual interest in sensory aspects of the environment (Mayes, Black, \& Tierney, 2013; Regier, Kuhl, \& Kupfer, 2013). Along with the changes in diagnosis, the classification of the disorders has also changed. The new diagnostic manual removed Asperger Syndrome and PDD-NOS as independently listed conditions, and instead replaced them with a sole diagnosis of Autistic Spectrum Disorder (AspergersAdvice, 2013).

In addition to the DSM V, there are three main instruments that clinicians and researchers may choose for early screening of Autism: The Checklist for Autism in Toddlers (CHAT), the modified CHAT (M-CHAT) and the Social Communication Questionnaire (SCQ) (Johnson \& Myers, 2007; Matson, Wilkins, \& Gonzalez, 2008). The CHAT consists of 14 items related to joint attention and pretend play; parents report on 9 items, while the 5 remaining are directly observed by the provider. The M-CHAT, a 23-item, yes/no parent questionnaire, was developed as a primary screening tool for autism at health supervision visits for children between 16 and 30 months of age. The SCQ, considered to be the most reliable, is a parent-report screen that comprises 40 yes/no questions. There are two forms of this assessment, one for children younger that six years and one for children aged seven years or older (Al-Qabandi, Gorter, \& Rosenbaum, 2011). 


\subsubsection{Prevalence and Causes}

\subsubsection{Occurrence}

The steady increase in the prevalence of ASD in the population between 2002 and 2013 is of much concern. As per statistics published by the center for disease control $(C D C)$, the overall prevalence was about 1 in 110 in 2006, which was a sharp increase from the finding reported in 2002, 1 in every 150 children. Furthermore, between 2007 and 2011-2012, the prevalence estimate for parentreported ASD diagnoses among American children aged 6-17 increased significantly, from $1.16 \%$ (or 1 in 86 ) to $2.00 \%$ (or 1 in 50 ). Interestingly there is also a biased in terms of the male to female ratio, with ASD being 5 times more common in males (Blumberg, Bramlett, Kogan, Laura, Jones, \& Lu, 2013; Centers for Disease Control and Prevention, 2012). There may be multiple factors contributing to the increase in prevalence, including the heightened awareness among pediatricians and parents, as well as genetic and epigenetic vulnerability, and even increasing maternal age (Sandin, Hultman, Kolevzon, Gross, MacCabe, \& Reichenberg, 2012).

ASD affects people of all socioeconomic and ethnic background, and isolating a specific risk factor for ASD is also challenging due to the substantial heterogeneity in the onset of autism. In cases of early-onset ASD, children have signs of developmental delays within the first 18 months of life; however, $25 \%-40 \%$ of children with autism initially demonstrate near-normal development until 18-24 months, then they regress and begin showing symptoms of autism. The possibility that there is early-onset versus regressive phenotypes of autism might have important implications for the types and degree of severity of ASD symptoms one 
may expect to encounter (Amaral, Schumann, \& Nordahl, 2008). Despite the absence of proven causes of autism, there are several hypothesis and theories regarding the potential mechanism of ASD symptoms manifestation

\subsubsection{Triple Hit Hypothesis}

A widely recognized theory regarding the large variability of symptoms observed in ASD is the Triple Hit Hypothesis. The hypothesis proposes that multifactorial disorders, such as ASD, will manifest symptoms only when all factors are active to different degrees and have overcome a certain threshold. The three factors mentioned are: exogenous stressors, an underlying vulnerability, and the stage of development when these insults and abnormalities take place (Casanova, 2007).

When considering potential exogenous factors, and environmental effects, there are several possibilities to consider. Environmental factors such as teratogens, including thalidomide and valproic acid are more likely to play a role in utero via maternal factors. It is also possible that maternal illness, such as rubella among many others, during pregnancy may contribute to the development of the disorder (Arndt, Stodgell, \& Rodier, 2005). Recently, the possible association between fetal testosterone concentration and certain autistic behaviors such as abnormal social relationships and restricted interests was also suggested (Johnson \& Myers, 2007). The results of a longitudinal study indicated that elevated fetal testosterone levels were positively associated with higher number of autistic traits in children ages 6-10 years (Auyeung, Baron-Cohen, Ashwin, Knickmeyer, Taylor, \& Hackett, 2009) 
The second potential factor referred to in the triple-hit hypothesis is the potential role of genetics; though several genes are linked to the various symptoms of autism, none have been directly related to the disorder (Rubenstein \& Merzenich, 2003). However, the disproportionate ratio of ASD affected males versus their female counterparts maybe a strong indicator of the association between sex chromosomal defects and ASD (Jamain, et al., 2003). Lastly, when considering the third factor of stage of brain development, many of the ASD abnormalities are associated with migratory and synchronization issues during prenatal development. Therefore, it would be logical to theorize that the origin of many ASD issues may lie in the first and second trimesters of pregnancy.

\subsubsection{Neurobiology and Neuropathology}

Neuroanatomical abnormalities in individuals on the spectrum may range from minor focal dysplasias, to heterotopias and more severe dysfunctional neural tissue irregularities related to corticogenesis. Dysplasias are a malformation of cortical development and are frequently associated with pediatric neurological disorders. Heterotopias, a type of cortical dysplasia resulting from altered neuronal migration, are abnormal distribution of gray matter nodular masses with disorganized or rudimentary lamination in the cortical or subcortical areas of the brain. Consequences of heterotopias may include, seizures cerebral palsy and severe intellectual developmental disabilities (Bailey, et al., 1998; Wegiel, et al., 2010).

Although abundant evidence of increased head circumference, brain weight and brain volume in autism, especially in childhood, exists; the underlying biological mechanisms of brain enlargement remain to be determined. These mechanisms 
could involve increased neurogenesis, gliogenesis, and/or synaptogenesis, as well as disturbed migration of neurons, decreased apoptosis and synaptic pruning or it may be a complex combination of these events (Bauman \& Kemper, 2005; Palmini, et al., 2004).

\subsubsection{Head Circumference}

Researchers have reported differences in head circumference $(\mathrm{HC})$ as well as brain volume between patients on the spectrum and their control counterparts at birth and during childhood; however similar studies in adolescence and adults on the spectrum have yielded none or insignificant difference between ASD and neurotypical (Hardan, Libove, Keshavan, Melhem, \& Minshew, 2009). In their large sample size, Sacco et al. (2007) reported macrocephaly as one of the most prominent neuroanatomical difference in 241 ASD patients 3-16 years of age compared to control individuals in the general population. Macrocephaly is defined as a head circumference being above the $97^{\text {th }}$ percentile on a normal growth curve chart. Their data conclusively showed that the distributions of height, weight, and head circumference in autistic patients do indeed differ significantly from those present in the general population (Sacco, et al., 2007).

A meta-analysis of more than a dozen magnetic resonance imaging (MRI) studies revealed that the brain enlargement in autism is time specific, and limited to ages 2-4 years (Redcay \& Courchesne, 2005). In their study regarding HC in ASD, Courchesne et al (2003) found that $76 \%$ of the children on the spectrum had $\mathrm{HC}$ measurements below the 50th percentile at birth. Surprisingly, $88 \%$ of the same sample group showed early and accelerated postnatal growth with HC 
measurements exceeding the 87 th percentile by 6 to 14 months, and $59 \%$ showed extreme increases during the first year (Courchesne, et al., 2001; Courchesne, Carper, \& Akshoomoff, 2003). The accelerated growth in ASD is followed by premature arrest of growth (Aylward, Minshew, Field, Sparks, \& Singh, 2002), which lets the neurotypicals to catch-up and therefore allowing adult ASD patients to similar head size as their age-matched neurotypicals. This process may provide an explanation for the differences reported in the neuropathology of ASD patients in childhood and in adolescence.

Dementieva et al. (2005) also conducted a similar study and found that of the 251 participants, about $35 \%$ of ASD patients had an abnormally accelerated rate in early cranial development. Interestingly, autistic individuals with accelerated head growth in early childhood displayed higher levels of adaptive functioning and less social impairment (Dementieva, et al., 2005). This observation especially provides support to the adaptive nature of the human brain after a potential early trauma; and aligns with the belief about synaptic pruning and brain plasticity following a post-natal injury.

\subsubsection{Brain Volume}

Reports of neuropathological changes in brain weight of autistic children have also surfaced. In their 2001 study, Courchesne and colleagues found an increase in total brain volume of autistic patients compared to control individuals. In an MRI study, the researchers found that the brain size of autistic toddlers was $10 \%$ greater in volume than normal and $90 \%$ of the autistic toddlers had volumes exceeding normal average (Bailey, et al., 1998; Courchesne, et al., 2001; Courchesne \& Pierce, 
2005). Post-mortem studies comparing brain weights of autistic cases to normative data have reported a high incidence of megalencephaly (brain weight greater than two standard deviations above the mean); one reported megalencephaly in $14 \%$ (Lainhart, et al., 1997) of autism cases examined and another in $75 \%$ (Bailey, Luthert, Bolton, Le Couteur, Rutter, \& Harding, 1993). Much of these differences have been attributed to the methodology utilized. For example, when the brain is weighed at autopsy, cerebrospinal fluid (CSF) in the leptomeninges is included in the weight measurement, which may lead to an erroneous overestimation of actual brain weight (Redcay \& Courchesne, 2005).

Neuroimaging studies have also shown similar trends in increased brain size in autistic children (Carper, Moses, Tigue, \& Courchesne, 2002; Courchesne, et al., 2001; Sparks, et al., 2002). The advantage of in vivo structural MRI studies lies in its ability to provide measurements of total brain volume excluding cerebrospinal fluid (CSF). However, a disadvantage is that the methodology of MRI studies can vary substantially on several dimensions, including type of scanner, segmentation programs, and anatomical boundary definitions. Furthermore, due to the late age of diagnosis, MRls are not ordinarily obtainable during the first two years of life because a clinical diagnosis of autism is often not made until the age of 18 months (Redcay \& Courchesne, 2005).

When discussing the neuropathology of adult ASD patients versus children on the spectrum: unlike the conclusive results regarding younger ASD patients, mixed results have been reported in adults and adolescents. Compared with control subjects, adolescent and adult patients on the spectrum have been reported as having either slightly increased (Hardan, Muddasani, Vemulapalli, Keshavan, \& 
Minshew, 2006; Hardan, Libove, Keshavan, Melhem, \& Minshew, 2009) or normal (Carper, Moses, Tigue, \& Courchesne, 2002; Courchesne, et al., 2001; Sparks, et al., 2002; Townsend, et al., 2001) brain volume. This is concurrent with the results reported of MRI studies regarding brain size. Maximum brain size was reached in autism by about 3-5 years of age, which is about 6 to 8 years earlier than normal, while neurotypical brains follow a typical head growth rate before plateauing at about 10 years of age (Carper, Moses, Tigue, \& Courchesne, 2002).

\subsubsection{Minicolumns}

During development, a process known as corticogenesis forms the cerebral cortex, and one aspect of this multidimensional process involves the migration of various types of cells towards the outer cortex of the brain. Cells surrounding the ventricles are known as periventricular germinal cells. The germinal cells provide neuroblasts that eventually give rise to pyramidal neurons, which release the excitatory neurotransmitter glutamate. These neuroblasts migrate to the cortex guided by a radial glial scaffold (Casanova, et al., 2006; Poluch \& Juliano, 2007). Other neuroblasts, which give rise to the inhibitory interneurons release neurotransmitter gamma amino-butyric acid (GABA), arise from the lateral and medial ganglionic eminence and travel tangentially toward the cortical plate (Poluch \& Juliano, 2007). Typically, in the cerebral cortex, roughly $80 \%$ of the neurons are excitatory glutamatergic and $20 \%$ are inhibitory GABAergic neurons (Rubenstein \& Merzenich, 2003). It is crucial that these migrational processes take place in a

synchronized manner, otherwise several abnormalities, both histological and functional, manifest. 
Minicolumns are made-up of layer V pyramidal cells, clusters of Layer II and III cells, as well as associated interneurons. The minicolumnar core comprises of radially oriented arrays of pyramidal projection neurons giving rise to axonal and dendritic bundles (Casanova, et al., 2006). Surrounding mainly the periphery of the minicolumn, combinations of GABAergic interneurons provide diverse signaling properties that serve to dynamically modulate pyramidal cell inputs and outputs, which are required to perform area- and task- specific information processing (Casanova M. F., 2007).

Varying with brain regions, each minicolumn contains on the order of 80-100 pyramidal neurons radially aligned through cortical layers II-VI. Center-to-center spacing between minicolumns ranges from 30 and 80 microns $(\mu \mathrm{m})$. Interneurons situated in the peripheral neuropil comprise about $15 \%$ to $25 \%$ of the total neuron compliment and modulate activity throughout the minicolumn. The strong vertical flow of the inhibitory activity of interneurons around the core creates a curtain of inhibition surrounding the complex (Casanova M. F., 2007; Casanova, El-Baz, Vanbogaert, Narahari, \& Switala, 2010).

The Gray Level Index (GLI) is defined as the ratio of the area covered by Nissl-stained elements to unstained area in postmortem samples; it is an estimator of the density of cell soma within a given region that includes cell number as well as cell size (Casanova M. F. et al., 2002a). Results of a post-mortem study based on GLI indicated that minicolumns in the brains of autistic patients were both more numerous and narrower than neurotypical brains, hinting toward decreased nucleolar size and a reduction in neuropil space. However, ASD constituent neurons 
were also more dispersed than in controls, accounting for the unaffected cellular density (Casanova, et al., 2002b; Casanova, Buxhoeveden, \& Gomez, 2003; Casanova, et al., 2006). These findings are indicative of an increase in short connections in exchange of longer connection fibers between various brain regions. Reductions in long-distance connectivity would be particularly detrimental to higherorder social and communicative functions requiring coherent integration of information from multiple sensory and cognitive domains (Herbert, et al., 2003; Redcay \& Courchesne, 2005)

\subsubsection{Dysplasia and Atrophy}

As suggested by the triple hit hypothesis, the type, timing, and severity of environmental insults, or the nature of genetic mutation and its resulting abnormal gene product at different stages of brain development will likely influence the observed histopathological and behavioral abnormalities of ASD. In contrast to other types of malformations caused by incomplete morphogenesis, such as hypoplasia (underdevelopment of tissue or organs) and aplasia (failure of development or absence of tissue or organs), dysplasias are malformations caused by disorganized development. Cortical dysplasias often result from dysfunctional migration and abnormal synchronization between neurons during corticogenesis (Kirschstein, et al., 2003; Palmini, et al., 2004).

Cortical atrophy refers to the changes in cortical structures after their initial formation. Often, the presence of diminished cell size, loss of neurons, and demyelination of white matter tracts can be indicative of atrophic processes. There are mixed results reported relating cortical atrophy and ASD brains. While many 
groups have reported atrophic abnormalities in various brain regions (Bauman \& Kemper, 2005; Kemper \& Bauman, 1998; Miles \& Hillman, 2000; Palmen, Engeland, Hof, \& Schmitz, 2004), others have reported absence of atrophic changes associated with ASD pathology (Rapin \& Katzman, 1998).

When considering the cerebellar changes associated with ASD; dysplasias of the cerebellum display an abnormal pattern of folia and/or presence of heterotopic nodules (Patel \& Barkovich, 2002). Hypertrophic and atrophic changes are also most marked in the cerebellar circuits of autistic brains, and occur in association with an apparent congenital decrease in the number of neurons in the cerebellar cortex. The age-related changes in the neurons are observed in multiple areas of the brain of autistic individuals appear to be a prolonged process that extends from neuronal hypertrophy in childhood to atrophy in later adult life (Kemper \& Bauman, 1998).

\subsubsection{Seizures and Autism}

In general, a seizure is defined as an episode of abnormal electrical activity in the brain, which is followed by the physical findings or changes in behavior. Seizures are transient occurrence of signs and/or symptoms due to abnormal excessive or synchronous neuronal activity in the brain (Fisher, et al., 2005). Epilepsy is described by the occurrence of two or more environmentally unprovoked (e.g., trauma, infection, alcohol withdrawal) seizures of any type, usually within a 3-year period (Hara, 2007; Matson \& Neal, 2009).

In a retrospective follow-up study, Hara (2007) reported results regarding one hundred and thirty individuals with autistic disorder or atypical autism diagnosed in childhood, who were followed up over 10 years and evaluated almost every year up 
to $18-35$ years of age. Thirty-three patients of the follow-up group (25\% of the sample) exhibited epileptic seizures; twenty of the epileptics (61\%) exhibited partial seizures. Although $18 \%$ of the non-epileptic group did exhibit epileptic discharges on EEG, $68 \%$ of the epileptic group revealed epileptiform EEG findings before the onset of epilepsy (Hara, 2007). Similar results have also been reported by others (Bolton, Carcini-Rathwell, Hutton, Goode, \& Howlin, 2011; Rossi, Parmeggiani, Bach, Santucci, \& Visconti, 1995) Autistic children with seizures/epilepsy in general have lower IQ, less adaptive behavior, more emotional problems, and higher psychiatric drug use (Matson \& Neal, 2009). Several theories have been speculated and studied to explain the abnormally high prevalence of seizures in ASD; however most reports contain arguable results; thus detailed explanation for the potential cellular abnormality leading to seizures remains mostly unknown.

\subsubsection{Cortical Thickness}

Cortical thickness may differ not only among individuals, but also throughout various brain regions of one individual; and these differences may be influenced by age, development, and disease processes (Barta, Miller, \& Qiu, 2005). Changes in cortical thickness during normal development relate to cognitive changes as children and adolescents mature (Gogtay, et al., 2004). This supports the possibility of utilizing cortical thickness as a measurable indicator for prognosis of symptom severity in terms of cognitive abilities in autism.

An initial MRI study conducted by Hardan et al. (2006) supported previous postmortem findings of increased cortical gray matter and increased cortical thickness in autistic patients. They posited that increased gray matter volume and 
total brain size observed in autism might be related to abnormal cortical connectivity. They did not report a significant difference in total brain volume or IQ amongst the sample size of autistic patients and neurotypical participants (Hardan, Muddasani, Vemulapalli, Keshavan, \& Minshew, 2006).

A later study published by the same group in 2009, pointed out that findings of increased cortical thickness in autism are not consistent within the literature and that the difference in reports is likely due to age-related changes, justifying the increased cortical thickness in children, but not in adolescents or adults. To explore this possibility, they investigated cortical thickness in ASD individuals and controls subjects using MRI technology to set a baseline and did follow-up MRI scans for developmental comparison. Their results showed that ASD individuals presented with a significant decrease in gray matter volume and decreased cortical thickness compared to neurotypical controls, but when they controlled for Full Scale Intelligence Quotient (FSIQ) in their analysis, there were no differences between the groups (Hardan, Libove, Keshavan, Melhem, \& Minshew, 2009).

Weigel et al. (2010) conducted a cohort study involving 13 subjects, and have reported a few neuropathological changes in autistic brains. Some of the abnormalities reported in the post-mortem study include increased thickness of the subependymal cell layer, subependymal nodular dysplasia, abnormal growth of the dentate nucleus and dysplasia of the granule layer in the dentate gyrus (Wegiel, et al., 2010). Another such study by Hustler et al. (2007) also used MRI to evaluate cortical thickness of post-mortem brain tissue of ASD individuals and their age and gender matched controls. The group found that the overall difference was very 
insignificant and maybe due to the methods they were using; it was suggested that MRI measures could result in misplaced boundaries to differentiate between the white and gray matter border (Hustler, Love, \& Zhang, 2007), thereby yielding results that overestimate thickness, because some of the white matter is identified as part of the cortical gray matter.

To find better and more confident results regarding gray matter differences between autistic and neurotypical individuals, a dual approach study of combining both MRI and voxel based morphometry (VBM) techniques has been performed in a given sample of participants (Hyde, Samson, Evans, \& Mottron, 2010). MRI scans were obtained for all participants, and then a surface deformation algorithm was used to determine differences in cortical thickness. Hyde et al. (2010) did not report any whole-brain cortical thickness differences; however they did find that the cortex was thicker in autism group in certain Broadman areas (BA) of the brain, frontal area (BA 9,10,11 and 44/45), temporal (BA 41), parietal (BA 39), and occipital (BA 17, 18 and 19) lobes. The VBM cortical gray matter results converged with the cortical thickness findings in terms of gray matter increases in middle and medial orbital frontal gyrus. The cortical thickness and VBM analyses also converged in terms of finding gray matter decreases in pre- and postcentral gyri, which is consistent with previous findings of gray matter differences in similar brain areas (Hyde, Samson, Evans, \& Mottron, 2010).

\subsubsection{Neuromorphology}

Reports of neuromorphological abnormalities in ASD cases are sparse, due to the limited number of post-mortem cases available, as well as the technical 
difficulties associated with the possible methodology. Santos et al (2011) reported that of the four postmortem cases of 4- to 11-year-olds with autism, approximately $53 \%$ had more spindle neurons in the anterior region of the cerebral cortex compared to the three controls (Santos, et al., 2011). In another small study, the investigators found $79 \%$ more neurons in the dorso-lateral prefrontal cortex and $29 \%$ more neurons in the mesial prefrontal cortex in seven brains of boys with autism compared with six brains of typically developing children; the size of the neurons were not determined (Courchesne, et al., 2011). Though these findings are concurrent with other abnormalities previously reported such as increased gray and white matter volume, the reliability of results are very arguable keeping in mind the very small sample size.

Besides the reports of a limited number of previously conducted studies, the cellular and molecular bases of pathology in the young autistic brain are largely unknown; however in the absence of direct evidence, speculations, inferred from autism symptomatology, have flourished. For example, among the numerous neuronal abnormalities that could lead to overgrowth in the young brain are accelerated and/or premature growth of neuron cell bodies, hyper-activate and enlarged glia, increased synapse and/or axon numbers, excessive and premature axonal and/or dendritic growth, as well as premature and excessive myelination (Bauman \& Kemper, 2005; Palmen, Engeland, Hof, \& Schmitz, 2004).

Another possible explanation regarding neuromorphological abnormalities is associated with the presence of seizures in ASD (Hussman, 2001; Kang \& Barnes, 2013). The researcher suggested that suppressed GABAergic inhibition is a common feature of the autistic brain. Since GABA is such an important inhibitory 
neurotransmitter, suppression of GABA producing interneurons may lead to a hyperexcitable brain environment, which may explain some of the typical symptoms in autism, including the manifestation of seizures (Hussman, 2001). 


\section{HYPOTHESIS}

\subsubsection{Cortical Thickness}

Based on previous studies about cortical abnormalities in ASD, as well as the associated symptoms, we hypothesize that the differences in cortical thickness will mainly be in the prefrontal cortex. Therefore concurring with the results of other studies regarding cortical thickness mentioned above.

Compared to past studies, the age range of our subjects was larger and symptom severity among the ASD patients also varied. Our study aims to address some of the previous limitations of other studies. Instead of looking at MRIs, we are investigating postmortem brain tissue, which will allow us to take a microscopic look at the areas of abnormal cortical thickness and manual segmentation allowed for better, more precise, delineation of gray matter from subcortical white matter.

\subsubsection{Neuronal Morphometry}

Keeping in mind the presence of ASD symptoms, such as seizures, we hypothesize that within the prefrontal cortex of ASD brain: there will be an increase in the excitatory pyramidal neurons, a decrease in the GABAergic inhibitory neurons,

or both abnormalities occurring simultaneously. Furthermore, the microscopic cortical abnormalities will be associated with errors specifically related to the synchronization between the radially migrating pyramidal neurons and the 
tangentially migrating interneurons. Hence, we believe that these changes may occur during the first and second trimester, since that is when a majority of neuronal migration and differentiation takes place. 


\section{METHODS AND MATERIALS}

\subsubsection{Subjects}

The postmortem tissue for this study was retrieved from the Autism Celloidin Library (ACL) of the Autism Tissue Program (ATP), which is the largest permanent brain tissue resource for neurological studies in autism. We studied seven agematched cases, which included individuals of a wide age range and cause of death. The source of our clinical data was the medical records of the autistic subjects, which consisted of psychological, behavioral, neurological and psychiatric evaluation reports. Table 1 outlines the presence or absence of seizures, medication history and cause of death.

The ATP obtained all of the records after the subjects' deaths. A certified rater and trainer arranged a visit with the family to obtain, with written consent, medical and clinical information via a questionnaire that included the Autism Diagnostic Interview-Revised (ADI-R). The ADI-R was administered as a standardized assessment tool in order to confirm the diagnosis on a postmortem basis. Inclusion of the subject in this study was based on a summary of scores of four domains: (a) qualitative abnormalities in reciprocal social interaction; (b) qualitative abnormalities in verbal and nonverbal communication; (c) restricted, repetitive and stereotyped patterns of behavior; and (d) abnormality of development evident at or before 36 months. The supporting documents such as autopsy reports, 
death certificates, medical, clinical, and/or educational records were obtained at the time of the home visit or by sending written requests, signed by the legal next-of-kin, to named providers.

The information obtained includes: donor and respondent identifying information; ethnicity, handedness and known exposure to hazardous materials; diagnostic information including dates and physician; genetic tests; pre- and postnatal medical history; immunization, medication, and hospitalization information;

\begin{tabular}{|c|c|c|c|c|c|c|}
\hline & ATP case \# & Age & Sex & Seizures & Medication history & Cause of death \\
\hline & Autism spect & a disor & & & & \\
\hline 1. & UMB-1627 & 5.0 & $\mathrm{~F}$ & $\mathrm{~N}$ & no medication & Multiple injuries / Motor vehicle accident \\
\hline 2. & AN13961 & 7.5 & M & $\mathrm{Y}$ & Phenobarbitol & Drowning \\
\hline & & & & & Tegretol & \\
\hline & & & & & Albuterol & \\
\hline 3. & AN00754 & 13.- & M & $\mathrm{Y}$ & Trileptol & Epilepsy \\
\hline & & & & & Trazadone & \\
\hline 4. & IBR-425-02 & 4.2 & $\mathrm{M}$ & $\mathrm{N}$ & asthma medication NOS & Drowning, Hypothermia \\
\hline 5. & AN19511 & 8.- & M & $\mathrm{N}$ & Peridex & Rhabdomyosarcoma \\
\hline & & & & & Nystatin & \\
\hline & & & & & GCSF & \\
\hline & & & & & Benadryl & \\
\hline & & & & & Pheergan & \\
\hline & & & & & Dexamethasone & \\
\hline & & & & & Morphine & \\
\hline 6. & AN02338 & 17.2 & $\mathrm{~F}$ & $\mathrm{~N}$ & Paxil & Cardiac arrest / Dilated cardiomyopathy \\
\hline 7. & AN09730 & 22.9 & M & $\mathrm{Y}$ & Neurontin & Choking / Epilepsy \\
\hline & & & & & Thioridazine & \\
\hline & & & & & Zonogran & \\
\hline & & & & & Fish oil & \\
\hline & & & & & Flaxseed oil & \\
\hline & & & & & CoEnzyme Q10 & \\
\hline & Neurotypical & & & & & \\
\hline 1. & UMB-1499 & 4.5 & $\mathrm{~F}$ & $\mathrm{~N}$ & N/A & Myocarditis \\
\hline 2. & UMB-4898 & 7.7 & $\mathrm{M}$ & $\mathrm{N}$ & N/A & Drowning \\
\hline 3. & BTB-3638 & 14.3 & M & $\mathrm{N}$ & N/A & Electrocution \\
\hline 4. & AN02456 & 4.- & $\mathrm{F}$ & $\mathrm{N}$ & N/A & Bronchopneumonia / Post-surgical procedure \\
\hline 5. & UMB-1708 & 8.1 & $\mathrm{~F}$ & $\mathrm{~N}$ & $\mathrm{~N} / \mathrm{A}$ & Multiple injuries \\
\hline 6. & UMB-1843 & 15.9 & $\mathrm{~F}$ & $\mathrm{~N}$ & N/A & Multiple injuries / Motor vehicle accident \\
\hline 7. & UMB-1646 & 23.2 & M & $\mathrm{N}$ & N/A & Rupture of spleen \\
\hline
\end{tabular}

Table 1 
family history and additional information about donor participation in any training or research studies such as imaging, medication trials, and/or genetic studies.

\subsubsection{Cortical Thickness}

\subsubsection{Tissue Acquisition}

Tissue for this study was retrieved from the Autism Tissue Program's Series 1 Autism Celloidin Library $(\mathrm{ACL})$, which is the largest permanent brain tissue resource for neurological studies in autism consisting of 42 age- and sex-matched whole hemispheres from both autistic individuals and neurotypical control subjects. Donor brains were removed and shipped to the Harvard Brain Tissue Resource Center within hours after death. Each brain was bisected at the midsagittal line along the longitudinal fissure into left and right hemispheres. For the Series 1 collection, each whole hemisphere was cut into coronal slices at $200 \mu \mathrm{m}$ thick and spaced $1.2 \mathrm{~mm}$ apart, then Nissl-stained, and permanently mounted and cover-slipped on slides. Shipments containing every sixth slide were then shipped to our lab at the University of Louisville for the present work. 


\subsubsection{Image Processing}

Tissue slides were imaged at 800 dpi (1pixel= $317.5 \mu \mathrm{m}$ on a Microteck ScanMaker i900 6400 × 3200 DPI CCD transparency, flatbed scanner with 48 Bit Color, capable of handling transparent media for wide-field, lowresolution imaging of tissue
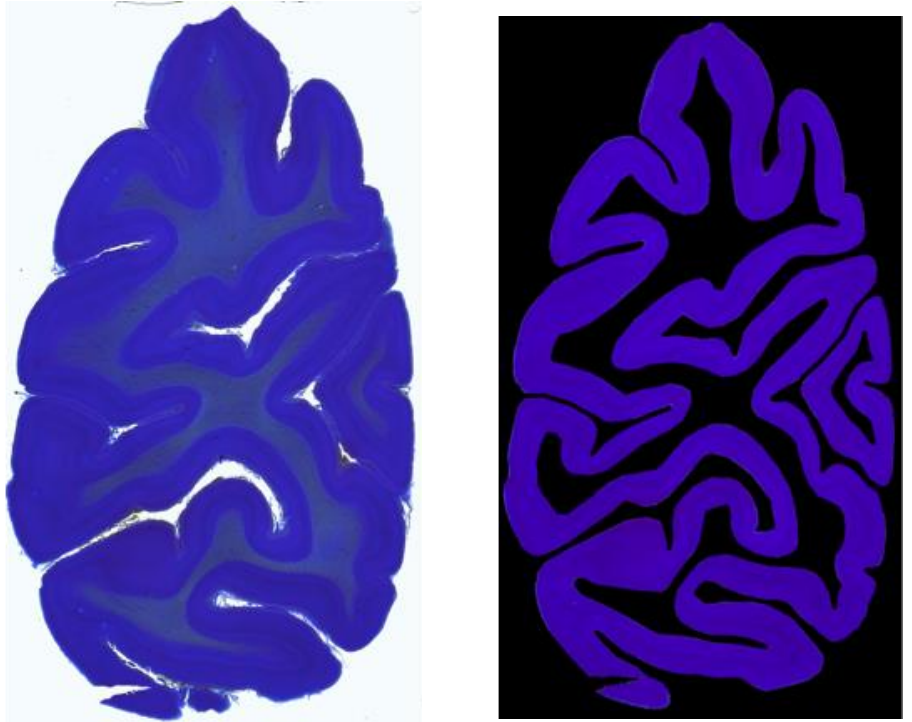

Figure 1: Imaged tissue slice (Left), and Segmented image (Right)

(Jones, Buchbinder, \& Aharon, 2000) to establish a digital library of each slide. Images were downloaded onto a Dell Precision workstation with two quad-core Intel Xeon processors and 24 GiB RAM per processor, running the latest version of Matlab for image processing and analysis, and $\mathrm{R}$ for statistical computation. The imaged tissue of the cerebral cortex was then manually segmented and converted into a binary image (Figure 1).

\subsubsection{Laplace Equation}

Cortical thickness was estimated using an algorithm based on electrostatics (Jones, Buchbinder, \& Aharon, 2000). Differences in cortical thickness between autistic and control subjects, were investigated by applying the Laplace equation to

measure the bandwidth from the inner to the outer boundary of the cortex ribbon in each slice throughout the entire hemisphere for each subject. 
Matlab software was used to solve the Laplace equation inside the cerebral cortex, which gave us the potential $(\varphi)$ that dictates the medial surface, $\varphi=0$. The outer contour, or the lamina I/lamina II boundary was set as $\varphi=-1$ and the inner contour, or the subcortical white matter boundary, was set as $\varphi=+1$.

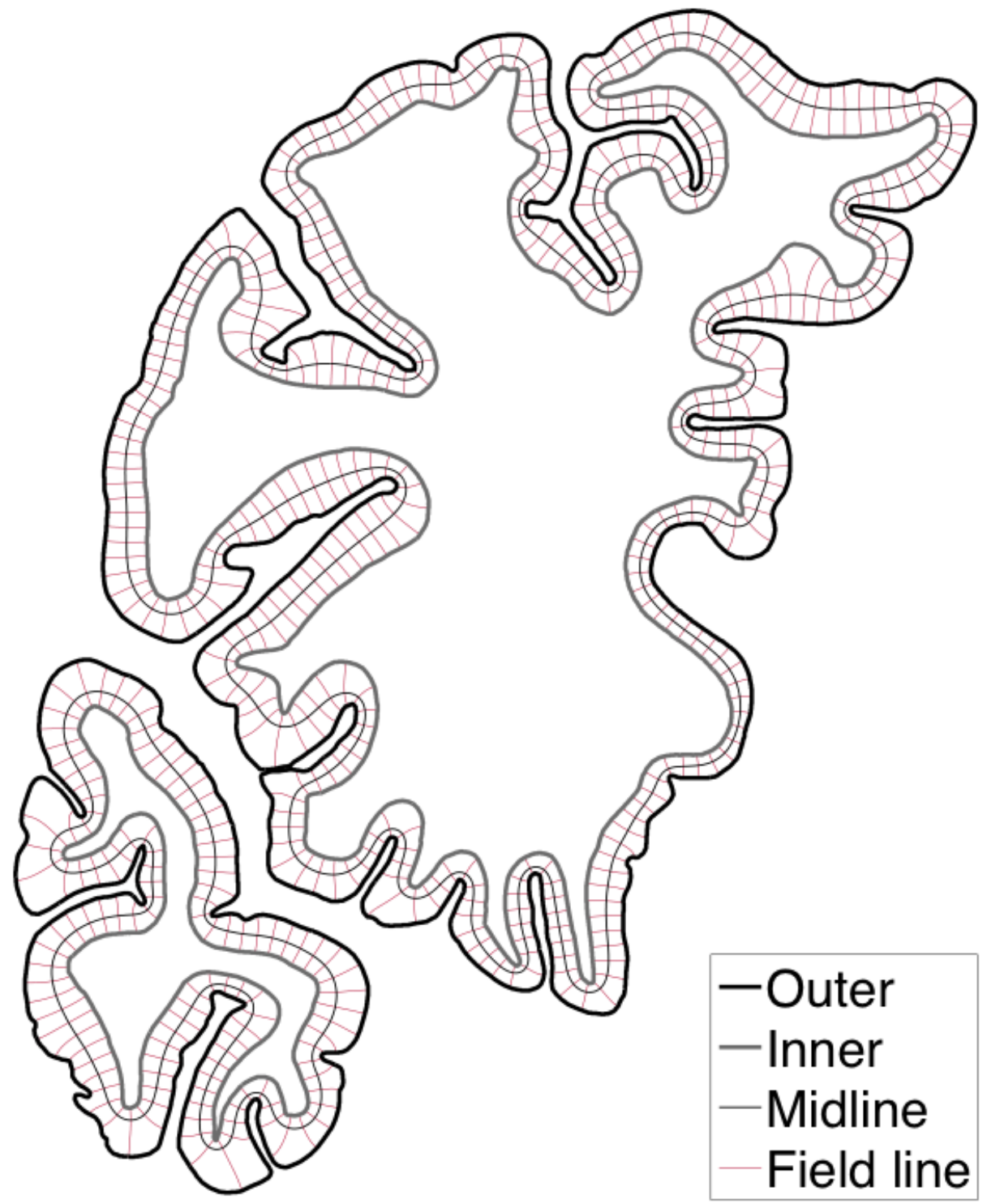

Figure 2: Solution of the Laplace equation inside the cortical ribbon. 
In addition to the medial surface, the equation yields field lines, which are the curves tangent to the gradient of the potential. The thickness (t) of the cortex at a point is then given by the length of the field line through that point, where field lines are defined as curves extending from the inner surface to the outer, everywhere parallel to $\nabla \varphi$ (Figure 2). Once the field lines were measured, they were binned according to their curvature and position along the cortical ribbon. In Figure 2, the field lines are displayed spaced at $1 \mathrm{~mm}$ intervals along the midline.

\subsubsection{Statistics}

The measurements were taken along the equipotential surface $\varphi=0$ in order that the thickness ( $t$ ) would be sampled evenly along the length of the cortical ribbon. Dysplastic regions were identified by first matching individual slides between ASDneurotypical pairs according to stereotaxic coordinate $y$. Coronal slices were positioned in MNI152 space so that $y=-106 \mathrm{~mm}$ at the occipital pole, $y=73 \mathrm{~mm}$ corresponds with the frontal pole, and the anterior commissure has position of $y=0$. The sampling distribution of $t$ in each slide from the neurotypical case was used to estimate "fences" beyond which statistical outliers would fall, using the method of Hubert and Van der Veeken. Regions of the corresponding slide in the matched ASD case where $t$ fell below the lower fence were identified as dysplasias.

A spine-smooth graph based on the cortical thickness results was created. The distance between inner and outer cortical profiles, or thickness of the cortex ( $\left.t^{\prime}\right)$, was plotted along the rostro-caudal axis. Variation in $\mathrm{t}^{\prime}$ with $\mathrm{x}$ was modeled nonparametrically using smoothing splines, with the degree of smoothing selected automatically by cross validation. Modified residuals from the smooth were 
resampled with replacement to generate artificial datasets, each of which was smoothed using the same procedure. These provided bootstrap estimates of the pointwise $95 \%$ confidence intervals on $\mathrm{t}^{\prime}(\mathrm{x})$.

A curvature versus position graph was also obtained, where the local curvature $(\kappa)$ of the brain surface was calculated on the same equipotential surface. Along the vertical axis, $\kappa=+1.5 \mathrm{~mm}^{-1}$ corresponded with the crest of the gyri, while $\kappa$ $=-1.5 \mathrm{~mm}^{-1}$ represents the base of the sulci. The horizontal axis represents the position of the coronal slides in a rostro-caudal direction; wherein the far left, or $70 \mathrm{~mm}$ is the frontal pole, $0 \mathrm{~mm}$ is the $A C$, and the far right, or $-106 \mathrm{~mm}$, is the occipital pole (Figure 5).

\subsubsection{Neuronal Morphometry}

\subsubsection{Neurolucida and Stereoinvestigator}

Once dysplastic regions were identified, we further analyzed twelve dysplastic regions from two ASD donor's brain. These regions were contrasted with twelve homologous regions from their respective neurotypical donors. In order to accomplish this, high-resolution images $(1$ pixel $=0.74 \mu \mathrm{m})$ of the regions of interest, spanning the cortex, were acquired.

The photomicrographs (Figure 3) were obtained utilizing the Neurolucida microscope along with the stereoinvestigator software. The Neurolucida workstation, consisted of an upright microscope configured to image in transmitted light, DIC, and epifluorescence modes (Nikon, Tokyo, Japan), a Dell Precision P-3500 desktop computer with Xeon processor (Dell, Round Rock, TX, USA) and Neurolucida 
software (MicroBrightField, Williston, VT, USA). Delineations were performed with a 1X- objective $(N A=0.04)$. Digital micrographs were produced using a $20 \mathrm{X}$ objective $(N A=0.40)$. Several such images were captured per region of interest to cover the area of the whole cortical ribbon. These images were assembled into one mosaic using the Virtual Slice module of the Stereoinvestigator software. Only slight adjustments of contrast and brightness were made, without altering the data of the original materials.

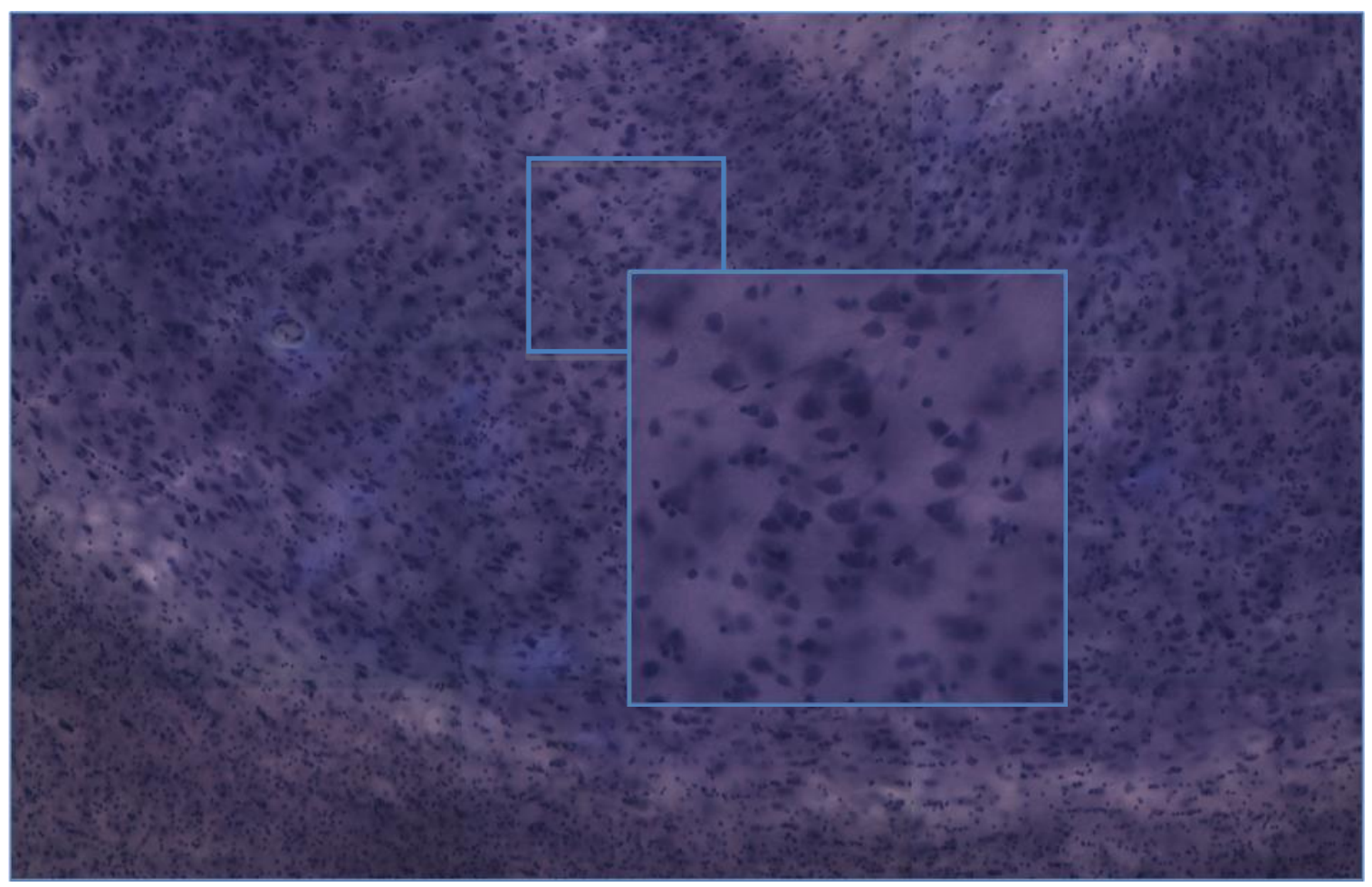

Figure 3: Photomicrograph of dysplastic region in ASD brain at 20X

\subsubsection{Segmentation Method}

Once the micrographs were obtained, pyramidal cells in the regions of interest were segmented following a three-step process. First, we employed a pixel- 
wise relaxation based on a maximum a posteriori (MAP) estimate of a pair-wise energy function of a generalized, two-dimensional Gauss-Markov random field (GGMRF) probabilistic model in order to reduce the effect of image noise (Casanova M. F., El-Baz, Vanbogaert, Narahari, \& Trippe, 2009). Secondly, we used a markerbased watershed approach to get an initial segmentation of the neurons from the Markov-Gibbs random field (MGRF) -relaxed image, obtained in the first step. Finally, for each initially segmented neuron, we refined its segmentation based on a local iterative thresholding approach. The optimum threshold is obtained in a way that maximizes the distance between the average signal intensities of the initially segmented neuron region and its background. Segmented binary images were analyzed using Boolean spatial statistical model and also granulometry.

\subsubsection{Boolean Model and Granulometry}

In order to investigate cell size patterns and abnormalities, we adopted the Boolean model, which is a model in stochastic geometry that yields explicit formulae that link characteristics on the particles to characteristics on the union of the particles. Statistics of the Boolean model began with the estimation of the mean values (mean area, volume, perimeter, etc.) of the Minkowski functionals of the grain (Molchanov, 1995).

Each realization of the Boolean model is the union of a number of convex random closed sets (grains)-independently and identically distributed with mean area, and mean perimeter located, or centered, at the points of a Poisson process with a given intensity; these three parameters completely determine the model (Casanova, et al., 2006). In the following equation used to establish the Boolean 
model, area occupied by Nissl-stained tissue $\left(A_{A}\right)$, the total boundary length of stained tissue per unit area $\left(\mathrm{L}_{\mathrm{A}}\right)$, and the planar convexity $\left(\mathrm{N}^{+}{ }_{\mathrm{A}}\right)$ were measured to compute the mean area $(\mathbf{A})$ and mean perimeter $(U)$ located, or centered, at the points of a Poisson process with intensity $(\lambda)$.

$$
\begin{gathered}
\mathbf{A}_{A}=\mathrm{e}^{-\lambda A} \\
\mathrm{~N}^{+}{ }_{\mathrm{A}}=\lambda\left(1-\mathrm{A}_{\mathrm{A}}\right) \\
\mathrm{L}_{\mathrm{A}}=\mathrm{N}^{+} \mathrm{U}
\end{gathered}
$$

The mathematical granulometric method, discovered by Georges Matheron, provides a morphological method for characterizing granular images by means of how they are sieved according to size and shape (Matheron, 1975). Pixels comprising a binary image are "sieved" according to their connectivity to similar pixels imposed by a certain primitive geometric body termed: structuring element. An integral characteristic of granulometry is the distribution of the pixels with respect to the diameters or radii of the original structural elements used. By exploiting this property, images can be simplified substantially to successfully isolate various classes of objects, also known as grains. This method can also act as a band-pass filter, which is capable of discriminating grains based not only on the size of the grain, but also on their similarity to a structuring element. By exploiting this property, images can be simplified substantially to successfully isolate various classes of objects (Dougherty \& Chen, 2001; Prodanov, Heeroma, \& Marani, 2006). 


\section{RESULTS}

An important element highlighted by our findings is that brain tissue in ASD is not atrophied; rather, it is dysplastic. Tissue atrophy is indicated by the presence of gliosis or other such cellular reactions that lead to inflammation; where as dysplasias, or malformations, exhibit neuronal disorganization or abnormal cell size. The cellularity within the molecular layer was judged as normal. The neuronal cytoarchitecture studied by microscopy confirmed our hypothesis regarding the dysplastic processes that occur in the brain of ASD patients. The absence of gliosis and the presence of normal cellularity both confirm that in ASD pathology, the tissue does not atrophy, but actually fails to develop normally.

\subsubsection{Inter-rater Reliability}

To control for quality and consistency in manual segmentation, five slides were randomly selected, then traced by three different individuals in order to determine inter-rater reliability. These findings indicate that the brains were manually segmented with an 87.1 percent aggregate inter-rater reliability.

\begin{tabular}{ll} 
Image & AC1 (\%) \\
425-02R19(1).tif & 82.3 \\
425-02R25(1).tif & 77.2 \\
425-02R43(1).tif & 89.6 \\
425-02R607(17).tif & 90.3 \\
425-02R85(2).tif & 90.3 \\
\hline - Aggregate) & 87.1
\end{tabular}




\subsubsection{Differences in Cortical Thickness}

The anterior commissure (AC) was taken as an anatomical landmark for the prefrontal cortex. Considering $t$ as a function of $y$ alone, mean cortical thickness was reduced in ASD in the vicinity of, and somewhat anterior to the AC. Six out of seven ASD patients showed diminutions within prefrontal cortex as defined by the AC. Figure 4 depicts the average thickness, in millimeter, for each slide plotted with respect to the slide's corresponding position in stereotaxic space. A significant reduction in mean thickness $(\mathrm{t})$ is visible near the occipital pole where findings were driven primarily by results in one patient; the slides of the said region in that patient also contained some visible artifacts. Consequently, the major dip in the occipital region maybe the result of an outlier, and an inaccurate indicator of the extent of cortical abnormalities in the given region. 


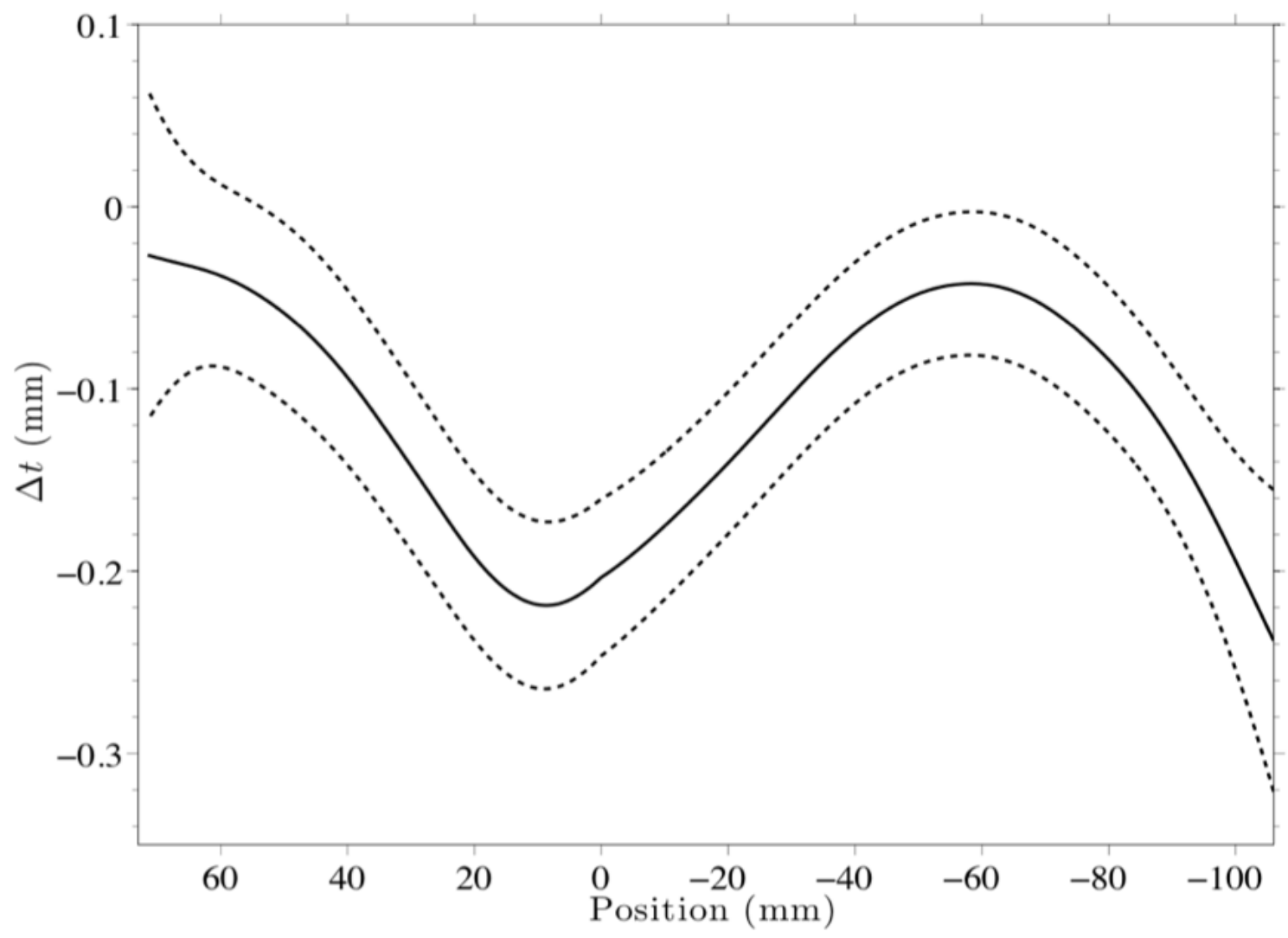

Figure 4: Spline smooth graph of cortical thickness results

Measurements taken over the cortex of neurotypical individuals were set as the baseline or normal $(\Delta t=0 \mathrm{~mm})$, and any value that fell two standard deviations above or below this baseline was considered to be significant. It is apparent that surrounding the AC, the overall width of the cortex in ASD brains is much thinner in comparison to their neurotypical control individuals. 
Figure 5 is based on the cortical thickness data averaged across the sample pairs. The horizontal axis represents the position of the given thickness in the cortex in relation to the AC. The left vertical axis represents curvature $(\kappa)$ : positive values are associated with the crest of the gyri and negative with the base of the sulci of the given region. As per the right vertical axis of the graph, the regions of ASD brains

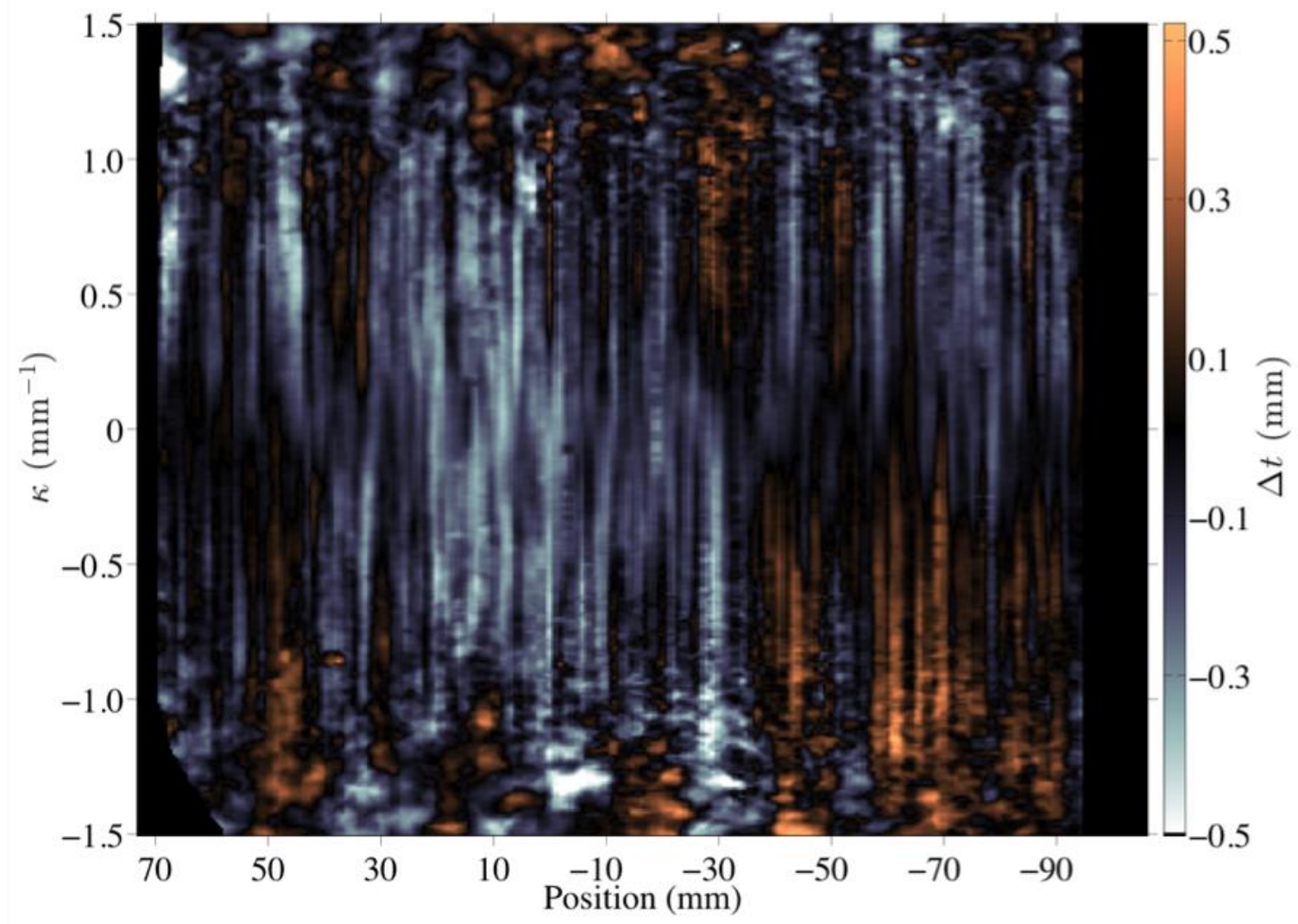

Figure 5: False Color Image, cortical thickness data were averaged across all seven pairs

found to be narrower than neurotypical are colored blue, while regions of atypically thicker cortex are colored orange. In general, the trend of differences in thickness according to the brain regions are noticeable; while the regions surrounding the $A C$, especially anterior to it, seem to be thinner, the frontal and occipital pole seem to be 
comparatively thicker. More specifically there are differences based on the curvature of the cortex; the negative curvature, or the base of the gyri, of the occipital pole was more thick than any other region, while the analogous area of the frontal pole is also thicker when compared to the regions surrounding the AC.

We elected to focus on the base or valley of the sulci, and not on the crest of the gyri, because inconsistencies in slicing the tissue or artifacts, such as variability in the angle at which the tissue was sliced, would affect the crest of the gyri while the base of the valley of the sulci would remain relatively unchanged.

\subsubsection{Neuronal Morphometry}

The results of the Boolean Model revealed an apparent trend in terms of the differences between ASD and neurotypical brain both by mean cell area as well as the perimeter. The method yielded results by lamina and the differences between the ASD and neurotypical also varied throughout the different laminae. We did not take into account the first lamina due to possibility of artifacts formed during tissue processing and mounting.

\subsubsection{Boolean Model: Mean Area and Mean Perimeter}

As per the results of the Boolean model (Figure 6), the mean cell area of the autistic patients was generally smaller than that of the control individuals across all laminae, II-VI. The differences were fairly consistent with the areas of greatest

difference being in lamina III and lamina VI. The greatest difference was in lamina III, wherein the median value of cell area of Autistic patients was $73 \mu \mathrm{m}^{2}$ in comparison to $90 \mu \mathrm{m}^{2}$ in neurotypical individuals. A large difference was also observed in lamina 
$\mathrm{IV}$, where the median cell area differed by approximately $15 \mu \mathrm{m}^{2}$; the cell area of the ASD tissue was smaller in comparison to neurotypical.
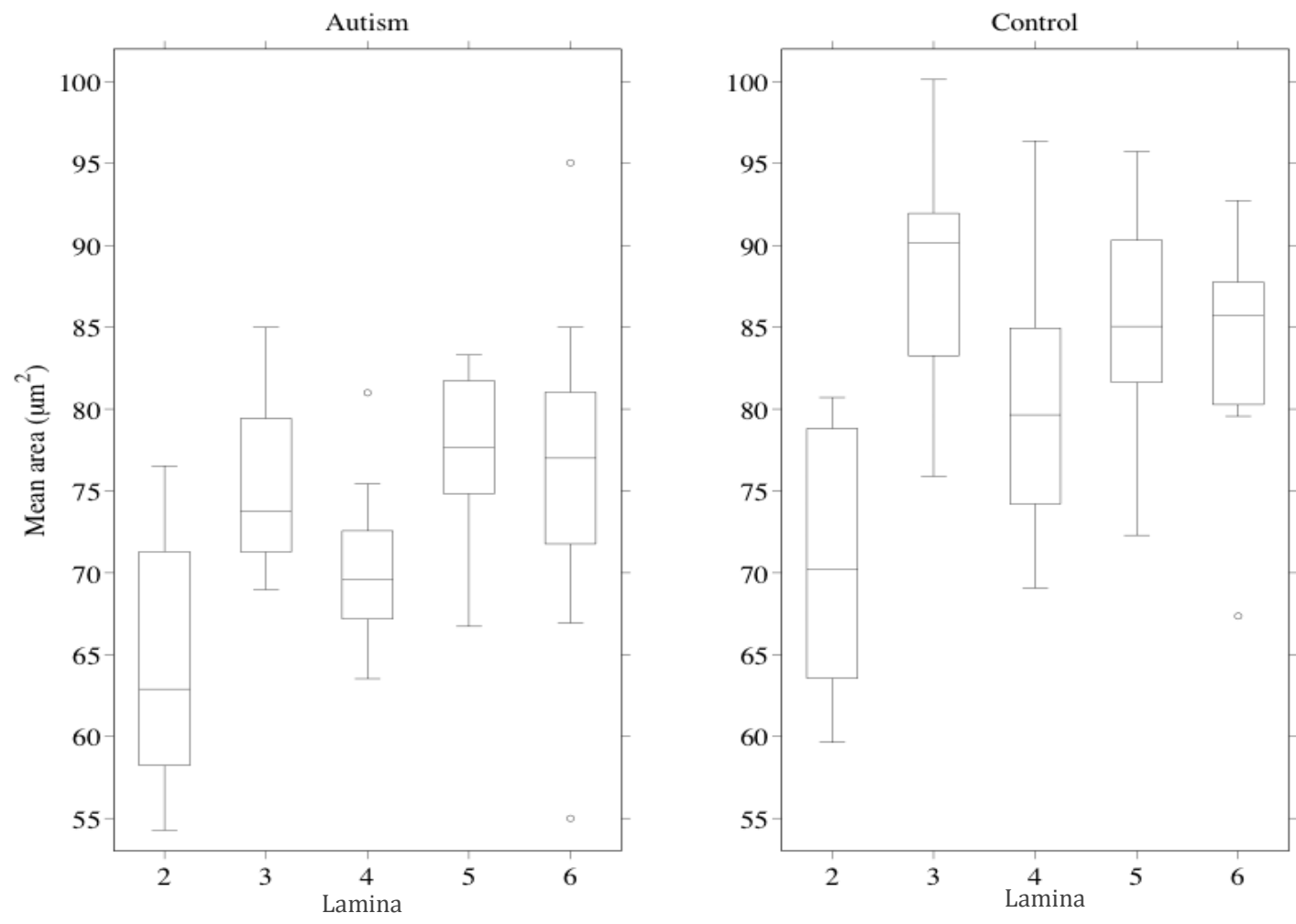

Figure 6: Box Plot of mean area of cells by lamina. ASD (Left) vs Control (Right)

Similar to the results of the mean cell area, the perimeter of various neurons also differed across the laminae, with the perimeter of cells in the ASD brain generally being smaller than of the control patients (Figure 7). Interestingly the differences were not as apparent as with the mean cell area, wherein the laminae in ASD being smaller by approximately $2 \mu \mathrm{m}^{2}$. 

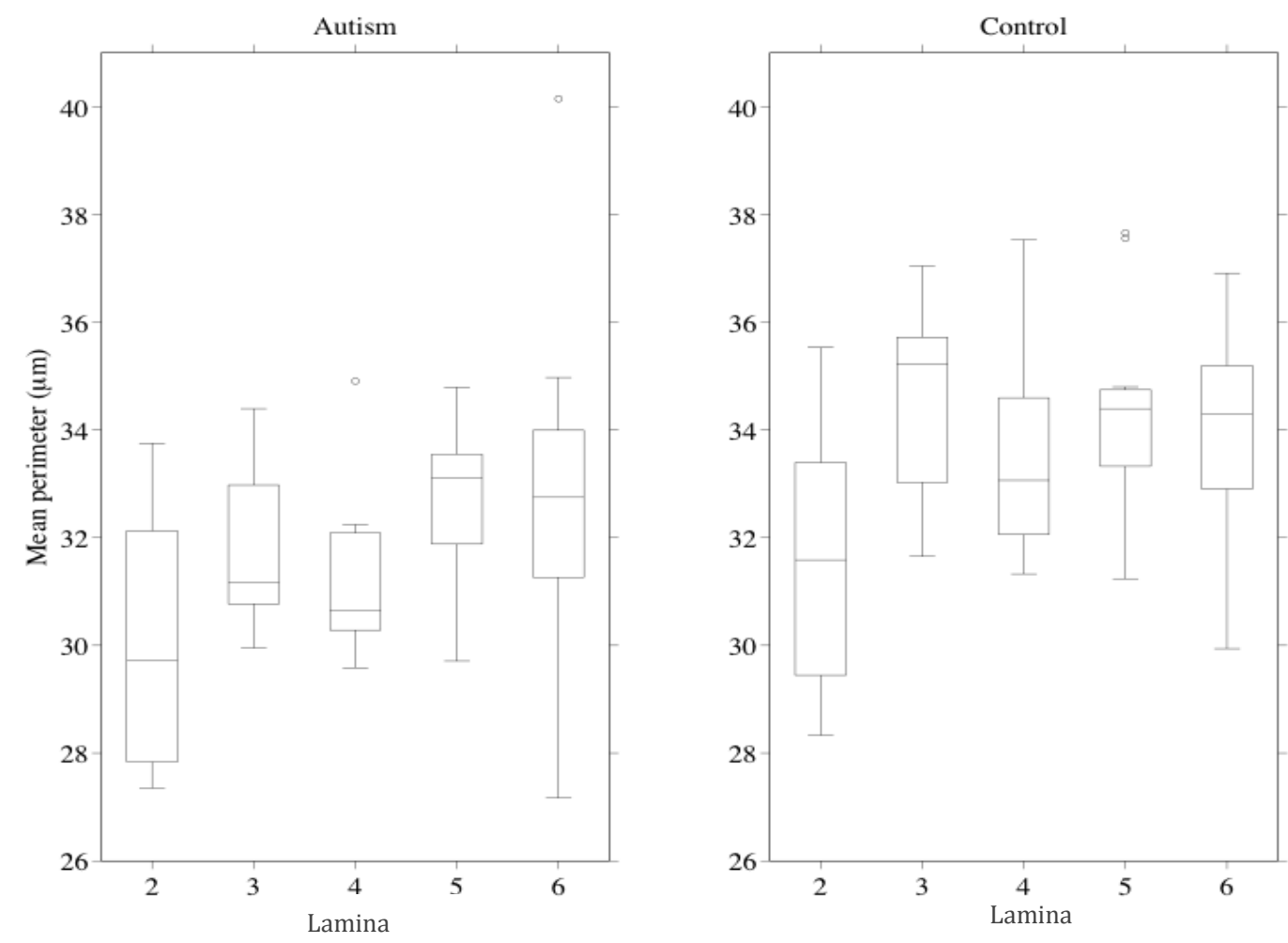

Figure 7: Box Plot of mean perimeter of cells by lamina. ASD (Left) vs Control (Right)

\subsubsection{Granulometry}

We utilized granulometry in order to investigate the specific neuronal differences among ASD and neurotypical brains. Figure 8 is a histogram of cell sizes by lamina obtained by granulometric analysis. The $y$-axis is arbitrary, since the actual stained area varied on the size of the region, only the relative differences are considered to be of importance. The x-axis, in micrometers, represents the radius of the structuring element. As per the graphs, there are remarkable differences in the neuronal size and laminar composition in the brains of ASD patients compared to their matched neurotypical individuals. Especially in laminae 3 and 6, where the 
volume of larger cells in the neurotypical brains is higher in comparison to ASD brains. In the same two laminae, the volume of smaller and medium neurons was higher in ASD brain; suggesting that the neuronal density in the cortices of ASD and neurotypical to be similar. In other words, although the pyramidal neurons are
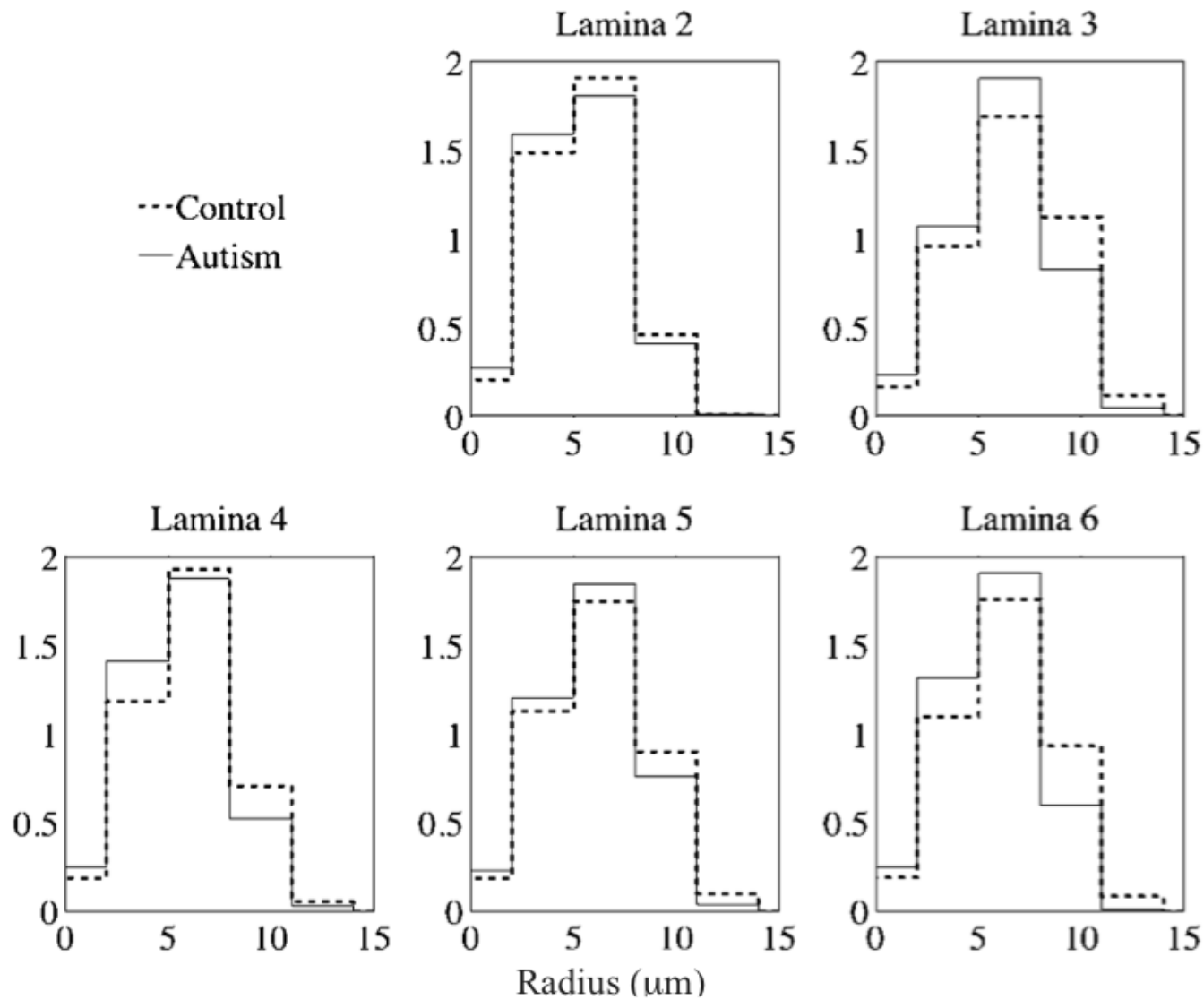

Figure 8: Pattern spectrum by lamina, obtained by granulometry on segmented (binary) images

smaller in ASD, they are numerous thus accounting for the normal neuronal density previously observed in ASD pathology. Additionally, the granulometry further confirms our finding of smaller neuron areas by the Boolean model. 


\section{DISCUSSION}

Autism spectrum disorders are a group of multidimensional disorders; common problems observed are disruptions associated with behavior, emotional interactions as well as, language and cognition. Among these defects, autistic individuals appear to have a range of perceptual processing abnormalities, expressed especially strikingly by a hypersensitivity to auditory and tactile stimuli (Rubenstein \& Merzenich, 2003). Researchers examining various aspects of how the autistic brain differs from neurotypical brains have used many advanced informatics software tools and examined research data of many types. These include visual neurologic data types such as post-mortem MRI, digitalized stained hemibrain sections, GLI techniques to measure cortical minicolumns architecture (Casanova, Buxhoeveden, \& Brown, 2002a; Casanova M. F., Buxhoeveden, Switala, \& Roy, 2002b), and three-dimensional reconstructions of stained serial sections (Bobinski, de Leon, \& Wegiel, 2000).

Some typical MRI research focuses on relative brain region size (Schumann, Buonocore, \& Amaral, 2001; Schumann et al., 2004), stereologic and detailed visual data relevant to design-based stereology (Schmitz \& Hof, 2005) as well as data related to the distribution of neurotransmitter receptors in various brain regions (Perry et al., 2001). Despite the abundance of research and many novel and intense techniques utilized, the specific etiology of the group of devastating deficits remains largely inconclusive. 


\subsubsection{Cortical Thickness and ASD}

We elected for a postmortem study, rather than neuroimaging, in order to optimize the resolution of the gray-white matter transition zone. Previous studies suggest that using neuroimaging to measure cortical thickness may provide for inaccurate results due to blurring of the transition zone, which could result in an overestimation of cortical thickness due to a misplaced lower boundary (Hustler, Love, \& Zhang, 2007). The overestimation could also lead to faulty speculation of increased number of neurons.

\subsubsection{Cortical Thickness and Initial Hypothesis}

The results of our study do support the initial hypothesis, as there are abnormalities through out many brain regions in $A S D$, and the most significant differences, as predicted, were found in the prefrontal cortex. Dysplastic foci, or cortical dysplasia, are abnormality where the neurons in an area of the brain failed to migrate and/or synchronize properly during corticogenesis. We observed multiple circumscribed dysplastic foci, and the described defects differed among affected individuals both in terms of location and overall size.

Cortical abnormalities followed a rostro-caudal pattern, with most defects being predominantly found at the crest of gyri near the pole regions and within the sulci and gyral crests towards the anterior commissure of the brain. Regions occupied small portions of gyri and seemed devoid of gross morphological abnormalities. Our data is in support of results published by another $2006 \mathrm{MRI}$ study 
that involved at 14 adult ASD patients and 14 closely matched neurotypicals. The group specifically found cortical thinning in certain regions of ASD brains, including areas belonging to the mirror neuron system, like the inferior frontal cortex, inferior parietal lobe and superior temporal sulcus, and of other areas involved in social cognition in individuals with ASD. Additional areas of cortical thinning were found in the prefrontal cortex, the anterior cingulate, the medial parietal cortex, and the supramarginal gyrus (Hadjikhani, Joseph, Snyder, \& Tager-Flusberg, 2006).

\subsubsection{Neuronal Morphometry and ASD}

Though various abnormalities have been reported speculating the morphology of the ASD brain, here we focused on the role of excitatory-inhibitory imbalance in autism pathology. More specifically, we aimed to study the two most important and abundant type of neurons that play a role in the excitation-inhibition balance in the human brain: the excitatory pyramidal cells, which secrete glutamate

and send myelinated axons outside the cortical mantle, and the inhibitory interneurons, which secrete GABA and are intrinsic to the cortex (Jones \& Peters, 1984; Vaccarino, Grigorenko, Smith, \& Stevens, 2009)

\subsubsection{Neuromorphology and Initial Hypothesis}

Together with measurement of cortical thickness and further investigation of the neuronal morphology, our lab has concluded that the dysplastic defects described in autism are very likely to result from disturbed cellular morphogenesis stemming from an interference with embryonic development. The dysplastic changes of the cerebral cortex coexist with heterotopias thus suggesting a plausible 
pathogenic mechanism in the division of periventricular germinal cells and consequentially, abnormalities in the ensuing migration of daughter cells to the cortex.

Our results of granulometric analysis indicated a reduction of larger cells and an overall increase in size of the smaller ones. A possible interpretation to the reported findings is the diminution in size of larger pyramidal cells and a reduction in total numbers of smaller neuronal elements (interneurons). The total reduction of the smaller, inhibitory, neurons and disturbed morphometry in general across the different cortical layers further suggests that once radially migrating neuroblasts reach the cortical plate they are desynchronized from the tangentially migrating interneurons.

\subsubsection{Neuromorphological Similarities}

Additionally, heterotopias and minicolumnar abnormalities are often observed in cases of idiopathic ASD; interestingly, other disorders exhibiting autism-like phenotype, such as congenital cytomegalovirus infection, antenatal cocaine exposure, extreme prematurity, tuberous sclerosis, and the Ehlers-Danlos syndrome, also share similar neuroanatomical abnormalities related to injuries to the germinal matrix during development. Therefore our findings, which indicate asymmetric division of periventricular germinal cells, may also be helpful to explain

neuropathological findings in many other disorders. 


\subsubsection{Significance of Frontal Lobe and Anterior Commissure}

The findings of abnormalities predominantly in the prefrontal lobe are not surprising since several ASD associated symptoms possibly originate from this region. It is recognized that individuals who have sustained damage to the frontal lobes have major executive function deficits, which are very similar to symptoms autistic patients experience. These include repetitive, aimless movements or speech, difficulty with self-regulation, tendency to hyper-focus on one aspect of information, impaired ability to act on or apply knowledge in a meaningful manner, and diminished capacity for planning ahead.

The behavior of patients on the spectrum often appears to be rigid and inflexible; many younger patients become distressed over trivial changes in the environment and insist on following routines in precise detail. They are often very persistent, focusing on one narrow interest or repetitively engaging in one stereotyped behavior. They may be impulsive, having trouble delaying or inhibiting responses (Fujii, Mori, Miyazaki, Hashimoto, Harada, \& Kagami, 2010). These functional issues that autistic and frontal lobe damage patients have in common provide firm evidence that frontal lobe pathology is one of the biggest contributors to autistic symptom, and our findings of the numerous dysplastic foci in the frontal lobe is concurrent with previous reports.

The prevalence of aggression is also astonishingly high in children and adolescents on the spectrum; a 2011 study revealed that in a large sample of ASD

patients, $56 \%$ were aggressive towards caregivers and $32 \%$ were aggressive towards others (Kanne \& Mazurek, 2011). Consistently, a more recent study has 
reported $53 \%$ of patients exhibiting aggressive behavior (Mazurek, Kanne, \& Wodka, 2013). Furthermore according to parental reports, $68 \%$ of ASD children had demonstrated aggression to a caregiver and $49 \%$ to non-caregivers. Bipolar disorder (BPD) is another disorder that is strongly associated with aggression. A recent study regarding the size of the anterior commissure $(A C)$ in BPD patients with aggression revealed the presence of a significantly smaller AC (Saxena, et al., 2012). These behavioral similarities could be correlated with the neuropathological findings of abnormal $A C$, and reduced cortical thickness in the same region. The AC connects the temporal lobes and amygdala of both hemispheres. Since the amygdala plays a role in the mediation of emotional processing, the mentioned findings were not completely unexpected. Though the findings of the study were about the white matter tract, our findings of thinner cortical width around the same region are pertinent since some of the tracts that makeup the $A C$ originate from the gray matter around the area. It is imperative to highlight this link between $A C$ diminution and aggression, as it may provide neuropathological origins of one of the most severe behavioral abnormalities that BPD and ASD patients share.

\subsubsection{Findings and Seizures in ASD}

Roughly $30 \%$ of autistic individuals develop clinically apparent seizures (Gillberg \& Billstedt, 2000), and $50-70 \%$ of autistic children have ongoing 'sharp spike' activity documented in sleeping EEG or magnetoencephalographic recording (Tuchman \& Rapin, 2002; Wheless, Simos, \& Butler, 2002). Our findings based on granulometry may lead to speculations regarding presence of seizures in ASD patients. A decrease in interneurons leads to decreased inhibition, which creates a 
hyper-excitable environment. Therefore a potential mechanism for the induction or propitiation of febrile seizures, a type of seizure associated with the presence of pyrexia or fever, could be attributed to this neuronal vulnerability of the brain combined with external triggers, such as increased body temperature. In neurotypical individuals, a fever would not usually generate a seizure, however in ASD this neuronal abnormality leaves the brain more susceptible to seizing when the body temperature increases.

\subsubsection{Cortical Thickness and Seizures}

The presence of multi-focal seizures and reduced cortical thickness has been reported, which compares to the results of the present study. These findings may lend to a potential explanation of the astonishingly high number of epileptic patients with the ASD population compared with the general population (Labate, et al., 2012). All seven autistic subjects in our study met ADI-R criteria for autism; three out of the seven autistic individuals suffered from seizures and six were on medication. None of the neurotypical patients had seizures and none were on any medications.

We attempted to make specific correlations between the abnormalities in certain brain regions with the presence or absence of seizure. However, because patients on the spectrum may have abnormal epileptiform discharges, without the manifestation of seizures EEG techniques are required to confirm abnormal electrical activity. Due to the lack of EEG reports and information regarding ASD patients without reported seizures, it would be difficult to make associations between cortical abnormalities and seizure occurrence. 


\subsubsection{Neuronal Morphometry and Seizures}

Seizures have long been associated with developmental abnormalities and neuronal imbalances. Various neurons have different sizes, shapes and purpose. Our study primarily focused on the potentially abnormal ratio between pyramidal neurons and interneurons. The differences between the two cells types are: pyramidal cells are much larger and their total dendritic length is longer compared to interneurons; the excitatory cells also receive several times more (two to ten times) synaptic inputs (nearly 32,000) than the inhibitory ones (about 3000-16,000), consistent with the fact that their total dendritic length is longer than for interneurons. Finally, it is important to note that in a neurotypical brain the ratio of inhibitory terminals on a pyramidal cell is generally lower than on interneurons(Megias, Emri, Freund, \& Gulyas, 2001).

The presence of seizures in a significant proportion of ASD cases is very likely to be the result of neuronal imbalance present in the brain of autistic individuals. Though reports have indicated the role of GABAergic suppression in the abnormal electrical activity of the ASD brain (Hussman, 2001), detailed explanation for the potential cellular abnormality leading to seizures remained mostly unknown. Our findings confirmed the presence of smaller pyramidal cells and suggested an overall reduction in the total number of interneurons in the ASD brain tissue, when compared to the neurotypical control. The presence of normal neuron density, despite the reduced size of the pyramidal neurons, does elude toward an increased number of neurons. In addition to the finding of granulometric analysis, our hypothesis that the size of the large neurons in the ASD brain would be diminished is 
further confirmed by the Boolean model results, in both area and perimeter (Figures 6 and 7). 


\section{CONCLUSIONS AND SUMMARY}

\subsubsection{Conclusions}

In conclusion, the results of our methods are supported and strengthened, directly or indirectly, by previous studies: (i) predominance of thinner minicolumns, and smaller cell size; (ii) increased white matter volume; (iii) initial accelerated head circumference growth.

(i) The anatomically identifiable minicolumns may represent a mechanism to integrate or segregate inputs and outputs of a group of cells (Mountcastle, 1978; Mountcastle, 1997). Post mortem analyses of the brains of autistic individuals revealed an increased number of anatomically identified cortical minicolumns with a higher packing density. There is an increase in column number in ASD patients, but the cellular density remains unaltered, which is suggestive of an increase in the number of excitatory neurons forming the minicolumns, or decreased volume or functionality of the inhibitory neurons surrounding the minicolumns, or all processes may be occurring at the same time (Casanova, Buxhoeveden, \& Brown, 2002a; Casanova, et al., 2006). These studies provide further support for our neuronal morphometric findings of the excitatory-inhibitory imbalance in ASD brains. In terms of 
minicolumnar development, abnormal cell migration and synchronization would decrease the distance between minicolumns; and the decreased distance means a decrease in GABAergic interneurons. Since GABA is the most prevalent inhibitory neurotransmitter, mutations or environmental factors that decrease GABA signaling would increase the brain's excitatory potential; and consequentially lead to or propitiate more seizures. Additionally, smaller and numerous minicolumns may lead to increased local connectivity, at the expense of decreased long-distance connections. All the local over-connectivity will also make it difficult for the brain to inhibit the electrical activity once it begins seizing.

(ii) Significant increases in both cerebral white and gray matter were found in children on the spectrum compared to the control group (normally developing, "typical" children), conversely, no such differences were reported in adolescent and adult ASD patients. (Akshoomoff, Pierce, \& Courchesne, 2002). This disparity between children and adults on the ASD spectrum has been investigated and loosely interpreted as a postnatal developmental abnormality. Myelination and axonal growth are substantial throughout childhood; in neurotypicals cerebral white matter volumes increase $59 \%$ between about 2-3 and 16 years of age (Courchesne, et al., 2001). This slow and methodical maturing of the brain does not happen in autism; instead, following the relatively brief period of overgrowth, there is a shift to reduced or arrested growth. Structures that had 
been far larger than normal in the 2- or 3-year- old autistic brain slow their growth rate very quickly. Another possibility to explain the abnormal white matter is a potential increase in pyramidal neuron number in ASD brains as well. Since the excitatory neurons send myelinated axons outside the cortex: an increase in the pyramidal neurons would mean more myelinated axons, which very nicely explains the increased white matter, and matches up perfectly with our predictions and findings.

(iii) Previous studies have already provided evidence of the abnormal and accelerated growth pattern of head size in ASD. At birth autistic patients have a normal or reduced brain size, however there is a rapid acceleration in brain growth, which gradually halts during toddlerhood, the growth in head size does resume and eventually plateaus during adolescence (Courchesne, Carper, \& Akshoomoff, 2003; Redcay \& Courchesne, 2005). When the brain grows too quickly, neurons do not have a chance to fully or properly mature, this is further supported by our findings of reduced size of certain neurons, which could mean underdevelopment. We observed an abundance of pyramidal neurons in ASD patients comparatively, which could also imply the failure of apoptosis during development. This excess could reflect defects in the regulation of neurogenesis, as well as delays and defects in apoptosis, perhaps involving glial dysfunction. 


\subsubsection{Neurodevelopment and ASD}

The findings of the present study, in combination with previous reports, may allude to the neurodevelopmental issues related to autism. Proliferation of cortical neurons is exponential between 10 and 20 weeks gestation and normally results in a net overabundance of neurons. The cortical plate, which gives rise to cortical layers, laminae II-IV, is formed by the $20^{\text {th }}$ gestational week, the formation occurs primarily by radially and tangentially migrating neurons (Kostovic, Judas, Rados, \& Hrabac, 2002; Prayer, et al., 2006). As per the findings of our study, this time during in utero development maybe the most vulnerable for ASD development, since majority of our findings indicate abnormalities associated with cell size and cell perimeter in all laminae of the ASD cortex. Additional support for this idea also includes the presence of the majority of anomalies in the prosencephalon, which is one of the first brain structures to develop after fertilization. The anterior portion of the brain begins to form before pregnancy is even detected; consequently this structure is also the most susceptible to injury. Once the basic building blocks of the human brain are damaged, it is extremely difficult for the development to progress normally, and the effects of the damage will almost always be apparent postnatally.

A potential or even probable explanation for seizures and brain overgrowth in ASD is very likely to be associated with abnormalities during the initial stages of corticogenesis. Early brain development involves two major, overlapping processes: 
progressive events and regressive events. The progressive events include neurogenesis, axon guidance, and formation of synapses and neurotransmitter receptors. The elimination processes, or regressive events, include normal programmed cell death (apoptosis), axon pruning, and synapse elimination (Akshoomoff, Pierce, \& Courchesne, 2002; Simonati, Tosati, Rosso, Piazzola, \& Rizzuto, 1999). The presence of increased number of neurons as well as the diminished size of the same would support the idea that: in autism, the brain maybe favoring progressive events over the regressive ones. In other words, it is possible that the rate of apoptosis is reduced and the rate proliferation is amplified, or that the two are co-occurring. Consequentially, neuron migration and synchronization will also suffer; and since specific neurons have specific tasks, such a problem in corticogenesis will have catastrophic effects on neurodevelopment.

All the prenatal developmental abnormalities mentioned lead to an excitatoryinhibitory imbalance of the cerebral cortex, which leads to a hyperexcitable environment. Postnatal maturational processes can amplify this defect, which can result in delayed synapse maturation or abnormal myelination, leading to the abnormal white matter in ASD brains previously reported. The white matter abnormality leads to the initial exponential brain overgrowth, which amplifies the sensory issues and heightened frequency of seizures experienced by ASD patients. It is difficult to isolate the precise starting point of this cascade of events in ASD pathology, however, it is fairly apparent that once this cascade begins it follows a positive feedback mechanism. 


\subsubsection{Strength of Present Study}

The strength of the present study lies in the combination of two methods: manual segmentation of tissue to study cortical thickness, and granulometric analysis of the dysplastic regions to quantify morphological differences. There are two main reasons that our methods may present more accurate and reliable results: in contrast to the MRI method, our method made the white and gray matter border somewhat more clear, and segmenting the post-mortem tissue manually further ensured the integrity of the gray matter measurements. We began by indentifying specific dysplastic areas of the ASD cortex in comparison to their age-matched neurotypical controls. We then examined the same tissue more in detail and identified the microscopic abnormalities. By studying each lamina we were able to more precisely infer the neurodevelopmental abnormalities in ASD. 


\section{LIMITATIONS AND FUTURE IMPLICATIONS}

\subsubsection{Number of subjects}

Currently, there are limited samples available for post-mortem study. To make more definite conclusions regarding the differences in cortical thickness, it would be ideal to have more tissue samples. Having further clinical data of the subjects, such as EEG reports and previous MRIs, maternal factors, and pregnancy history, would also prove to be helpful so more specific correlations could be made between the neuropathology and the symptomatology.

\subsubsection{Condition of the Tissue}

Artifacts within the tissue that occurred during the cause of death, fixation, staining, mounting on slides and the preservation of tissue over time could all affect the results of this study. For example, it is possible that some anomalies found were the result of the cause of death rather than a cause or result of autism. In regards to the fixation process, fixtures denature macromolecules by changing the shape of large molecules, fixatives produce morphological patterns, and the care given to handling the tissue during the fixation process could result in tearing of the tissue.

The intensity of the staining could be too dark or too light depending on whether or not the tissue fully absorbed the stain and whether or not the stain was absorbed equally throughout the slice of tissue. Mounting the tissue on slides could 
also result in tearing of the tissue. Too much mounting medium or any air bubbles incased between the cover slip and the slide could also contribute to distorted imaging of the tissue. Any tissue data that was determined to be unusable due to the state of its condition was not included in the statistical analysis and reported as deleted data, however, since this data needed to either be reconstructed or thrown out completely, it may have affected our statistical analysis.

\subsubsection{Unbiased Stereology in the Future}

Design-based stereology permits precise and reliable measurement of exact number, size and spatial distribution of cells within a given brain region, using standardized protocols. Unbiased, or design-based, stereological methods can be applied to materials that are inhomogeneous or cannot be assumed to be homogeneous (i.e. the various types of neurons in the cortex).

This method could not be pursued further in this study, mainly due to the thickness of the tissue used. At 200 microns, the various cell layers were overlapping, and despite using advance techniques, we were unable to overcome the issue. Based on previous studies, we believe the ideal thickness of the tissue for such a study would have been about 35 microns. 


\section{REFERENCES}

1. Akshoomoff, N., Pierce, K., \& Courchesne, E. (2002). The Neurobiological Basis of Autism from a Developmental Perspective. Development and Psychopathology , 14, 613-634.

2. Al-Qabandi, M., Gorter, J. W., \& Rosenbaum, P. (2011). Early Autism Detection: Are We Ready for Routine Screening? Pediatrics , 128 (1), e211e217.

3. Amaral, D. G., Schumann, C. M., \& Nordahl, C. W. (2008). Neuroanatomy of Autism. Trends in Neurosciences , 31 (3), 137-145.

4. Arndt, T. L., Stodgell, C. J., \& Rodier, P. M. (2005). The teratology of autism. International Journal of Developmental Neuroscience , 23 (2-3), 189-199.

5. Auyeung, B., Baron-Cohen, S., Ashwin, E., Knickmeyer, R., Taylor, K., \& Hackett, G. (2009). Fetal testosterone and Autistic traits. The British Psychological Society, 100, 1-22.

6. Aylward, E. H., Minshew, N. J., Field, K., Sparks, B. F., \& Singh, N. (2002). Effects of age on brain volume and head circumference in autism. Neurology , 59, 175-183.

7. Bailey, A., Luthert, P., Bolton, P., Le Couteur, A., Rutter, M., \& Harding, B. (1993). Autism and megalencephaly. Lancet, 341, 1225-1225.

8. Bailey, A., Luthert, P., Dean, A., Harding, B., Janota, I., Montgomery, M., et al. (1998). A Clinicopathological Study of Autism. Brain , 121, 889-905.

9. Barta, P., Miller, M. I., \& Qu, A. (2005). A Stochastic Model for Studying the Laminar Structure of Cortex from MRI. IEEE Transactions on Medial Imaging , 24 (6), 728-742. 
10. Bauman, M. L., \& Kemper, T. L. (2005). Neuroanatomic observations of the brain in autism: a review and future directions. International Jouran of Developmental Neuroscience , 23, 183-187.

11. Blumberg, S. J., Bramlett, M. D., Kogan, M. D., Laura, S. A., Jones, J. R., \& Lu, M. C. (2013). Changes in Prevalence of Parent-reported Autism Spectrum Disorder in School-aged U.S. Children: 2007 to 2011-2012. National Health Statistics Reports , 65.

12. Bobinski, M., de Leon, M. J., \& Wegiel, J. (2000). The Histological Validation of Post Mortem Magnetic Resonance Imaging- Determined Hippocampal Volume in Alzheimer's Disease. Neuroscience , 95 (3), 721-725.

13. Carper, R. A., Moses, P., Tigue, Z. D., \& Courchesne, E. (2002). Cerebral lobes in autism: early hyperplasia and abnormal age effects. Neurolmage , 16, 1038-1051.

14. Casanova, M. F. (2007). The Neuropathology of Autism. Brain Pathology, $17,422-433$.

15. Casanova, M. F., Buxhoeveden, D. P., Switala, A. E., \& Roy, E. (2002a). Neuronal Density and Architecture (Gray Level Index) in the Brains of Autistic Patients. Journal of Child Neurology , 17 (7), 515-521.

16. Casanova, M. F., Buxhoeveden, D., \& Brown, C. (2002b). Clinical and Macroscopic Correlates of Minicolumnar Pathology in Autism. Journal of Child Neurology , 17 (9), 692-695.

17. Casanova, M. F., Buxhoeveden, D., \& Gomez, J. (2003). Disruption in the Inhibitory Architecture of the Cell Minicolumn: Implications for Autism. Neuroscientist , 9 (6), 496-507.

18. Casanova, M. F., El-Baz, A., Vanbogaert, E., Narahari, P., \& Switala, A. (2010). A Topographic Study of Minicolumnar Core Width by Lamina Comparison between Autistic Subjects and Controls: Possible Minicolumnar Disruption due to an Anatomical Element In-Common to Multiple Laminae. Brain Pathology , 20 (2), 451-458.

19. Casanova, M. F., El-Baz, A., Vanbogaert, E., Narahari, P., \& Trippe, J. (2009). Minicolumnar width: Comparison between supragranular and infragranular layers. Journal of Neuroscience Methods , 184 (1), 19-24. 
20. Casanova, M. F., van Kooten, I. A., Switala, A. E., van Engeland, H., Heinsen, H., Steinbusch, H. W., et al. (2006). Minicolumnar Abnormalities in Autism. Acta Neuropathalogica, 112:, 287-303.

21. Centers for Disease Control and Prevention. (2012). Autism Spectrum Disorders (ASDs). Retrieved February23,2013, from http://www.cdc.gov/ncbddd/autism/data.html

22. Courchesne, E., \& Pierce, K. (2005). Brain Overgrowth in Autism During a Critical Time in Development: Implications for Frontal Pyramidal Neuron and Interneuron. Developmental Neuroscience , 23, 153-170.

23. Courchesne, E., Carper, R., \& Akshoomoff, N. (2003). Evidence of brain overgrowth in the first year of life in autism. JAMA , 290 (3), 337-344.

24. Courchesne, E., Karns, C. M., Davis, H. R., Ziccardi, R., Carper, R. A., Tigue, Z. D., et al. (2001). Unusual Brain Growth Patterns in Early Life in Patients with Autistic Disorder. Neurology, 57, 245-254.

25. Courchesne, E., Mouton, P. R., Calhoun, M. E., Semendeferi, K., AhrensBarbeau, C., Hallet, M. J., et al. (2011). Neuron Number and Size in Prefrontal Cortex of Children With Autism. Journal of American Medical Association , 306 (18), 2001-2010.

26. Dementieva, Y. A., Vance, D. D., Donnelly, S. L., Elston, L. A., Wolpert, C. M., Ravan, S. A., et al. (2005). Accelerated Head Growth in Early Development of Individuals With Autism. Pediatric Neurology, 32 (2), 102108.

27. DiCicco-Bloom, E., Lord, C., Zwaigenbaum, L., Courchesne, E., Dager, S. R., Schmitz, C., et al. (2006). The Developmental Neurobiology of Autism. The Journal of Neuroscience , 26 (26), 6897-6906.

28. Dougherty, E. R., \& Chen, Y. (2001). Robust optimal granulometric bandpass filters. Signal Proc , 81, 1357-1372.

29. DSM-V - What Does It all Mean? (2013, January 16). AspergersAdvice.org. Retrieved April 5, 2013, from http://www.aspergersadvice.org/dsm-v-whatdoes-it-all-mean/ 
30. Fischl, B., \& Dale, A. M. (2000). Measuring the thickness of the human cerebral cortex from magnetic resonance images. Proceedings of National Academy of Science , 97 (20), 11050-11055.

31. Fisher, R. S., van Emde Boas, W., Blume, W., Elger, C., Genton, P., Lee, P., et al. (2005). Epileptic Seizures and Epilepsy: Definitions Proposed by the International League Against Epilepsy (ILAE) and the International Bureau for Epilepsy (IBE). Epilepsia , 46 (4), 470-472.

32. Fujii, E., Mori, K., Miyazaki, M., Hashimoto, T., Harada, M., \& Kagami, S. (2010). Function of the frontal lobe in autistic individuals: a proton magnetic resonance spectroscopic study. The Journal of Medical Investigation , 57, 35-44.

33. Gillberg, C., \& Billstedt, E. (2000). Autism and Asperger syndrome: coexistence with other clinical disorders. Acta Psychiatica Scandinavica, 102, 321-330.

34. Gogtay, N., Giedd, J. N., Lusk, L., Hayashi, K. M., Greenstein, D., Vaituzis, A. C., et al. (2004). Dynamic mapping of human cortical development during childhood through early adulthood. Proceedings of the National Academy of Sciences of the United States of America , 101 (21), 8174-8179.

35. Hadjikhani, N., Joseph, R. M., Snyder, J., \& Tager-Flusberg, H. (2006). Anatomical Differences in the Mirror Neuron System and Social Cognition Network in Autism. Cerebral Cortex , 16, 1276-1282.

36. Hara, H. (2007). Autism and epilepsy: A retrospective follow-up study. Brain and Development , 29, 486-490.

37. Hardan, A. Y., Libove, R. A., Keshavan, M. S., Melhem, N. M., \& Minshew, N. J. (2009). A Preliminary Longitudinal MRI Study of Brain Volume and Cortical Thickness in Autism. Biological Psychiatry, 66 (4), 320-326.

38. Hardan, A. Y., Muddasani, S., Vemulapalli, M., Keshavan, M., \& Minshew, N. J. (2006). An MRI Study of Increased Cortical Thickness in Autism. Am J Psychiatry, 163 (7), 1290-1292.

39. Herbert, M. R., Ziegler, D. A., Deutsch, C. K., O'Brien, L. M., Lange, N., Bakardjiev, A., et al. (2003). Dissociations of Cerebral Cortex, Subcortical 
and Cerebral White Matter Volumes in Autistic Boys. Brain , 126, 11821192.

40. Hussman, J. P. (2001). Suppressed GABAergic Inhibition as a Common Factor in Suspected Etiologies of Autism. Journal of Autism and Developtmental Disorders , 31 (2), 247-248.

41. Hustler, J. J., Love, T., \& Zhang, H. (2007). Histological and Magnetic Resonance Imaging Assessment of Cortical Layering and Thickness in Autism Spectrum Disorders. Biological Psychiatry , 61, 449-457.

42. Hyde, K. L., Samson, F., Evans, A. C., \& Mottron, L. (2010). Neuroanatomical Differences in Brain Areas Implicated in Perceptual and Other Core Features of Autism Revealed by Cortical Thickness Analysis and Voxel-Based Morphometry. Human Brain Mapping , 31, 556-566.

43. Jamain, S., Quach, H., Betancur, C., Rastam, M., Colineaux, C., Gillberg, C., et al. (2003). Mutations of the X-linked Genes Encoding Neuroligins NLGN3 and NLGN4 are Associated with Autism. Nature Genetics , 34, 2729.

44. Johnson, C. P., \& Myers, S. M. (2007). Identification and Evaluation of Children With Autism Spectrum Disorders. American Academy of Pediatrics , 120, 1183-1215.

45. Jones, E. G., \& Peters, A. (1984). Cellular Components of the Cerebral Cortex (Vol. 1). New York: Plenum.

46. Jones, S. E., Buchbinder, B. R., \& Aharon, I. (2000). Three-Dimensional Mapping of Cortical Thickness Using Laplace's Equation. Human Brain Mapping , 11, 12-32.

47. Kanne, S. M., \& Mazurek, M. O. (2011). Aggression in Children and Adolescents with ASD: Prevalence and Risk Factors. Journal of Autism and Developmental Disorders , 41, 926-937.

48. Kemper, T. L., \& Bauman, M. (1998). Neuropathology of Infantile Autism. Journal of Neuropathology and Experimental Neurology , 57 (7), 645-652. 
49. Kirschstein, T., Fernandez, G., Grunwald, T., Pezer, N., Urbach, H., Blumcke, I., et al. (2003). Heterotopias, cortical dysplasias and glioneural tumors participate in cognitive processing in patients with temporal lobe epilepsy. Neuroscience Letters , 338 (3), 237-241.

50. Kostovic, I., Judas, M., Rados, M., \& Hrabac, P. (2002). Laminar Organization of the Human Fetal Cerebrum Revealed by Histochemical Markers and Magnetic Resonance Imaging. Cerebral Cortex , 12 (5), 536544.

51. Labate, A., Cerasa, A., Mula, M., Murmoli, L., Gioia, M. C., Aguglia, U., et al. (2012). Neuroanatomic correlates of psychogenic nonepileptic seizures: A cortical thickness and VBM study. Epilepsia , 53 (2), 377-385.

52. Lainhart, J. E., Piven, J., Wzorek, M., Landa, R., Santangelo, S., Coon, H., et al. (1997). Macrocephaly in Children and Adults With Autism. Journal of the American Academy of Child and Adolescent Psychiatry , 36 (2), 282290.

53. Matheron, G. (1975). Random sets of integral geometry (2nd ed ed.). New York: Wiley.

54. Matson, J. L., \& Neal, D. (2009). Seizures and epilepsy and their relationship to autism spectrum disorders. Research in Autism Spectrum Disorderss , 3, 999-1005.

55. Matson, J. L., Wilkins, J., \& Gonzalez, M. (2008). Early Identification and Diagnosis in Autism Spectrum Disorders in Young Children and Infants: How Early is Too Early? Research in Autism Spectrum Disorders , 2, 75-84.

56. Mayes, S. D., Black, A., \& Tierney, C. D. (2013). DSM-5 under-identifies PDDNOS: Diagnostic agreement between the DSM-5, DSM-IV, and Checklist for Autism Spectrum Disorder. Research in Autism Spectrum Disorders , 7, 298-306.

57. Mazurek, M. O., Kanne, S. M., \& Wodka, E. L. (2013). Physical Aggression in Children with Autism Spectrum Disorders. Research in Autism Disorders , 7, 455-465. 
58. Megias, M., Emri, Z., Freund, T., \& Gulyas, A. I. (2001). Total Number and Distribution of Inhibitory and Excitatory Synapses on Hippocampal CA1 Pyramidal Cells. Neuroscience, 102 (3), 527-540.

59. Miles, J. H., \& Hillman, R. E. (2000). Value of a Clinical Morphology Examination in Autism. American Journal of Medical Genetics , 91, 245-253.

60. Molchanov, I. S. (1995). Statistics of the Boolean Model: From the Estimation of Means to the Estimation of Distribitions. Advances in Applied Probability , 27, 63-86.

61. Mountcastle, V. B. (1978). An Organizing Principle for Cerebral Function: The Unit Module and The Distributed System. In G. M. Edelman, \& V. B. Mountcastle, The Mindful Brain: Cortical Organization and the GroupSelective Theory of Higher Brain Function (pp. 7-50). Massachusetts Inst of Technology Pr.

62. Mountcastle, V. B. (1997). The Columnar Organization of the Neocortex. Brain , 120, 701-722.

63. National Institute of Mental Health. (2011 йил 26-October). A Parent's Guide to Autism Spectrum Disorder. Retrieved 2013 йил 3-April from National Institute of Mental Health: http://www.nimh.nih.gov/health/publications/aparents-guide-to-autism-spectrum-disorder/what-is-autism-spectrumdisorder-asd.shtml

64. Palmen, S. J., Engeland, H. V., Hof, P. R., \& Schmitz, C. (2004). Neuropathological Findings in Autism. Brain , 127, 2572-2583.

65. Palmini, A., Najm, I., Avanzini, G., Babb, T., Guerrini, R., Foldvary-Schaefer, N., et al. (2004). Terminology and classification of the cortical dysplasias. Neurology , 62 (Suppl 3), S1-S8.

66. Patel, S., \& Barkovich, A. J. (2002). Analysis of Classification of Cerebellar Malformations. American Journal of Neuroradiology , 23, 1074-1087.

67. Poluch, S., \& Juliano, S. L. (2007). A Normal Radial Glial Scaffold Is Necessary for Migration of Interneurons During Neocortical Development. Glia , 55, 822-830. 
68. Prayer, D., Kasprian, G., Krampl, E., Ulm, B., Witzani, L., Prayer, L., et al. (2006). MRI of normal fetal bain development. European Journal of Radiology , 57, 199-216.

69. Prodanov, D., Heeroma, J., \& Marani, E. (2006). Automatic morphometry of synaptic butons of cultured cells using granulometric analysis of digital images. Journal of Neuroscience Methods , 151, 168-177.

70. Rapin, I., \& Katzman, R. (1998). Neurobiology of Autism. Neurological Progress , 43 (7), 7-14.

71. Redcay, E., \& Courchesne, E. (2005). When is the Brain Enlarged in Autism? A Meta-Analysis of All Brain Size Reports. Biological Psychiatry, $58,1-9$.

72. Regier, D. A., Kuhl, E. A., \& Kupfer, D. J. (2013). The DSM-5: classification and criteria changes. World Psychiatry, 12, 92-98.

73. Rubenstein, J. L., \& Merzenich, M. M. (2003). Model of Autism: Increased Ratio of Excitation/Inhibition in Key Nerual Systems. Genes, Brain and Behavior , 2, 255-267.

74. Sacco, R., Militerni, R., Frolli, A., Bravaccio, C., Gritti, A., Elia, M., et al. (2007). Clinical, Morphological, and Biochemical Correlates of Head Circumference in Autism. Biological Psychiatry, 62 (9), 1038-1047.

75. Santos, M., Uppal, N., Butti, C., Wicinski, B., Schmeidler, J., Giannakopoulos, P., et al. (2011). von Economo Neurons in Autism: A Stereologic Study of the Frontoinsular Cortex in Children. Brain Research , 1380, 206-217.

76. Saxena, K., Tamm, L., Walley, A., Simmons, A., Rollins, N., Chia, J., et al. (2012). A Preliminary Investigation of Corpus Callosum and Anterior Commissure Aberrations in Aggressive Youth with Bipolar Disorders. Journal of Child and Adolescent Psychopharmachology , 22 (2), 112-119.

77. Simonati, A., Tosati, C., Rosso, T., Piazzola, E., \& Rizzuto, N. (1999). Cell Proliferation and Death: Morphological Evidence During Corticogenesis in the Developing Human Brain. Microscopy Research and Technique , 45, 341-352. 
78. Sparks, B., Friedman, S., Shaw, D., Alyward, E., Echelard, D., Artru, A., et al. (2002). Brain structural abnormalities in young children with autism spectrum disorder. Neurology, 59, 184-192.

79. Townsend, J., Westerfield, M., Leaver, E., Makeig, S., Jung, T.-P., Pierce, K., et al. (2001). Event-related brain response abnormalities in autism: evidence for impaired cerebello-frontal spatial attention networks. Cognitive Brain Research , 11, 127-145.

80. Tuchman, R., \& Rapin, I. (2002). Epilepsy in Autism. The Lancet Neurology, 1, 352-358.

81. Vaccarino, F. M., Grigorenko, E. L., Smith, K. M., \& Stevens, H. (2009). Regulation of Cerebral Cortical Size And Neuron Number by Fibroblast Growth Factors: Implications For Autism. Journal of Autism Developmental Disorders , 39 (3), 511-520.

82. Wegiel, J., Kuchna, I., Nowicki, K., Imaki, H., Wegiel, J., Marchi, E., et al. (2010). The Neuropathology of Autism: Defets of Neurogenesis and Neuronal Migration, and Dysplastic Changes. Acta Neuropathologica, 119, 755-770.

83. Wheless, J. W., Simos, P., \& Butler, I. J. (2002). Language Dysfunction in Epileptic Conditions. Seminars in Pediatric Neurology , 9 (3), 218-228. 


\section{CURRICULUM VITAE}

NAME: $\quad$ Shweta Sunil Kamat

ADDRESS: 176 Hays Blvd

Lexington, KY 40509

sskama01@louisville.edu

859-519-8564

\section{EDUCATION:}

University of Louisville, Louisville, KY

Expected graduation: December 2013

\section{Master of Science}

Major: Anatomical Sciences and Neurobiology

Transylvania University, Lexington, $\mathrm{KY}$

May 2011

\section{Bachelor of Arts}

Majors: Psychology, Biology (Double Major)

University of Kentucky Sanders Brown Center on Aging

June-August 2010

\section{Research Internship}

Role: Laboratory Assistant

Kentucky Institute for International Studies

June-July 2009

\section{Study Abroad}

Program: Community and Consumer Studies in Denmark

\section{RESEARCH EXPERIENCE:}

\section{University of Louisville School of Medicine, Louisville, KY}

Graduate Thesis: Post-Mortem Study of Cortical Dysplasia in Autism.

Dr. Manuel Casanova (2012-Present)

Assisted: Effects of Ambient Prism Lens in Autism

Dr. Manuel Casanova (2012) 


\section{University of Kentucky Sanders Brown Center on Aging, Lexington, KY}

Undergraduate Research: Role of microglial activation in Alzheimer's disease.

Linda Van Eldik (2010)

Kentucky Institute for International Studies, Program: Denmark

Study Abroad Student of Community and Consumer Health Studies in Denmark. Institution: Murray State University (2009)

\section{POSTER PRESENTATION or ABSTRACTS}

\section{Poster Presentation:}

Kamat, S.S., Dombroski, B.A., McNutt, A.N., Switala, A.E., El-Baz A. S., Casanova, M. F. (2013). Post-Mortem Study of Variable Differences in the Cortical Thickness and Neuronal Morphometry of Autistic Individuals vs. Controls. Presented at 2013 Society for Neuroscience, Neuroscience Day University of Louisville, Louisville, KY

Kamat, S. S., Dombroski, B. A., Switala, A. E., El-Baz A. S., Casanova, M. F. (2012). Variable Differences in the Cortical Thickness of Autistic Individuals Compared to Controls. Presented at 2012 Society for Neuroscience, Neuroscience Day University of Louisville, Louisville, KY

\section{Abstract submission:}

Kamat, S.S., Dombroski, B.A., Allison-McNutt, A., Switala, A.E., El-Baz, A.S., and Casanova, M.F. 2013 International Meeting for Autism Research (IMFAR), Donostia/ San Sebatian, Spain. Abstract: Focal Cortical Dysplasias of the Cerebral Cortex in Autism.

\section{Publication:}

Casanova, M., El-Baz, A., Kamat, S., Dombroski, B., Khalifa, F., Elnakib, A., Soliman, A., Allison-McNutt, A., Switala, A. Focal Cortical Dysplasia in Autism Spectrum Disorders. Acta Neuropathologica Communications, 1(1), 67

\section{Awards and Recognition}

$2^{\text {nd }}$ place "Engineering Collaboration Award" at Research! Louisville 2012 Undergraduate: Deans List 